\title{
Homogenization of the degenerate two-phase flow equations
}

\author{
Patrick Henning, Mario Ohlberger, and \\ Ben Schweizer
}

Preprint 2012-04

April 2012

Fakultät für Mathematik

Technische Universität Dortmund

Vogelpothsweg 87

44227 Dortmund

$\underline{\text { tu-dortmund.de/MathPreprints }}$ 



\title{
Homogenization of the degenerate two-phase flow equations
}

\author{
Patrick Henning ${ }^{1}$, Mario Ohlberger ${ }^{1}$, and Ben Schweizer ${ }^{2}$
}

April 2, 2012

\begin{abstract}
We analyze two-phase flow in highly heterogeneous media. Problems related to the degeneracy of the permeability coefficient functions are treated with a new concept of weighted solutions. Instead of the pressure variables we formulate the problem with the weighted pressure function $\psi$, which is obtained as the product of permeability and pressure. We perform the homogenization limit and obtain effective equations in the form of a two-scale limit system. The nonlinear effective system is of the classical form in the non-degenerate case. In the degenerate case, the two-scale system uses again a weighted pressure variable. Our approach allows to work without the global pressure function. Even though internal interfaces are included, our approach provides the homogenization limit without any smallness assumptions on permeabilities or capillary pressures.
\end{abstract}

MSC: 76S05, 35B27

key-words: homogenization, two-phase flow, degenerate permeability

\section{Introduction}

Flow problems in porous media are usually modelled with time dependent partial differential equations. Unsaturated media are often described with the Richards equation, if two immiscible fluids must be modelled, then the two-phase flow system is commonly used.

\subsection{Two-phase flow in porous media}

We consider two immiscible fluids, one is denoted with a subscript $w$, the other with a subscript $n$. In the following, we use an index $j$ that stands for either $w$ or $n$, the

\footnotetext{
${ }^{1}$ Westfälische Wilhelms-Universität Münster, Center for Nonlinear Sciences, Einsteinstr. 62, D-48149 Münster, Germany.

${ }^{2}$ Technische Universität Dortmund, Fakultät für Mathematik, Vogelpothsweg 87, D-44227 Dortmund, Germany.
} 
letters are chosen in view of applications where one fluid is wetting, the other is nonwetting. The saturations of the two fluids are denoted by $s_{w}$ and $s_{n}$, the pressures of the two fluids by $p_{w}$ and $p_{n}$, the negative fluxes by $q_{w}$ and $q_{n}$. The description of the porous material consists in the choice of three coefficient functions: two permeability functions $k_{j}^{\text {tot }}$ and one capillary pressure function $p_{c}$. We write the two-phase flow system as

$$
\begin{aligned}
\partial_{t} s_{j} & =\nabla \cdot q_{j} \quad \text { in } \Omega, \text { for } j=w, n \\
q_{j} & =k_{j}^{\text {tot }}\left(s_{j}\right) \nabla p_{j} \quad \text { in } \Omega, \text { for } j=w, n \\
s_{w}+s_{n} & =1, \quad p_{w}-p_{n} \in p_{c}\left(s_{w}\right) \quad \text { in } \Omega,
\end{aligned}
$$

where we neglected the dependence on the time variable $t \in[0, T)$ in all equations and unknowns. We wrote the system in such a way that it contains the six unknowns $s_{j}, p_{j}, q_{j}$ (of which the fluxes are vector valued) and six equations (1.1)-(1.3) (of which (1.2) is vector valued). The equations are for incompressible fluids, gravity is neglected, porosity and densities are normalized.

In many applications, one assumes a multiplicative decomposition of the total permeability as $k_{j}^{\text {tot }}(x, s)=K_{j}(x) k_{j}(s)$, into an absolute permeability $K_{j}$ that depends only on the position $x$, and a relative permeability $k_{j}$ that depends only on the saturation $s$. We will make a similar, but slightly less restrictive assumption below, assuming that the relative permeability depends on macrosscopic position and on the saturation, but that it is, microscopically, piecewise constant.

Physically correct models for unsaturated media require the use of a multi-valued capillary pressure function in (1.3). This is a consequence of the fact that in regions without wetting fluid $\left(s_{w}=0\right)$, the non-wetting pressure $p_{n}$ can be increased arbitrarily; the analogous reasoning applies for $s_{w}=1$. We note that, even in the multi-valued setting, we can define a saturation function $\Theta=p_{c}^{-1}: \mathbb{R} \rightarrow[0,1]$, and the second relation of (1.3) may be written in the form $s_{w}=\Theta\left(p_{w}-p_{n}\right)$. In this formulation, we have to deal with flat parts in the monotone function $\Theta$. A crucial consequence is the following: in regions with $s_{w}=0$, a wetting pressure $p_{w}$ is physically not well-defined. Mathematically, we read relation (1.2) in regions with $s_{w}=0$ as the statement that also the negative flux $q_{w}$ must vanish.

Our approach uses ideas from [25]. The fundamental observation is that, even though $p_{w}$ is not well defined globally, the product $\psi_{w}:=k_{w} p_{w}$ is well defined by setting $\psi_{w}=0$ whenever $k_{w}=0$. In the corresponding concept of weighted solutions, we use the quantity $\psi_{w}$ instead of the pressure $p_{w}$.

\subsection{Modelling of heterogeneous media}

We use a parameter $\varepsilon>0$ to describe the small length-scale of the heterogeneity of the porous medium. We consider periodic media and denote the unit cell by $Y=[0,1)^{n}$, always imposing periodicity conditions on the cell boundaries. Oscillations of the coefficient functions $K_{j}, k_{j}$ and $p_{c}$ are prescribed by functions

$$
\begin{aligned}
& K_{j} \in L^{\infty}(\Omega \times Y, \mathbb{R}), \\
& k_{j}: \Omega \times Y \times[0,1] \rightarrow[0, \infty) \text { is given as } k_{j}(x, y, s)=k_{j}^{m}(x, s) \text { for } y \in Y_{m} \\
& \text { for } M \text { functions } k_{j}^{m}: \Omega \times[0,1] \rightarrow \mathbb{R}, m=1, \ldots, M .
\end{aligned}
$$




$$
p_{c}: Y \times[0,1] \rightarrow \mathbb{R} \text { is given as } p_{c}(y, s)=p_{c}^{m}(s) \text { for } y \in Y_{m}
$$

for $M$ functions $p_{c}^{m}:[0,1] \rightarrow \mathbb{R}, m=1, \ldots, M$.

Here, we decomposed the unit cell in subdomains as $\bar{Y}=\bigcup_{m=1}^{M} \bar{Y}_{m}$, such that the open subsets $Y_{m}$ are pairwise disjoint. The number $M \in \mathbb{N}$ denotes the number of different materials that are distributed in a fine mixture. We emphasize the important restriction in the above setting: the capillary pressure coefficient does not vary in an arbitrary fashion in $y$, but a $y$-independent coefficient function $p_{c}^{m}$ is used in the subdomain corresponding to $Y_{m}$. To describe the heterogeneous material, we use

$$
K_{j}^{\varepsilon}(x):=K_{j}\left(x, \frac{x}{\varepsilon}\right), \quad k_{j}^{\varepsilon}(x, s):=k_{j}\left(x, \frac{x}{\varepsilon}, s\right), \quad p_{c}^{\varepsilon}(x, s):=p_{c}\left(\frac{x}{\varepsilon}, s\right) .
$$

\subsection{Related literature}

Due to its importance in practical applications, there is a vast literature on flow problems in porous media. If the medium is completely saturated by one fluid, the flow is described by Darcy's law and incompressibility, and no deep mathematical theory must be used. When two immiscible phases are present in the medium, but one of the fluids need not be modelled (a constant pressure can be assumed), then one uses Richards equation. In this equation, existence questions are interesting due to the degeneracy of the permeability, see e.g. [3, 25, 27]. When both phases must be modelled, one typically uses the two-phase flow system that we investigate here. Again, the degenerate permeabilites make existence results intricate, compare $[13,14,20]$. Usually, existence results use the global pressure as an additional variable. The global pressure is defined in such a way that it solves an elliptic equation and estimates on the global pressure can be exploited in the further analysis. Nevertheless, there are situations in which the global pressure is of very limited use; this is the case e.g. in problems with hysteresis $[6,19]$ and in problems with outflow boundary conditions $[4,21,24]$.

Homogenization. The homogenization problem for two-phase flow equations receives considerable attention. This is due to the fact that the limit problem has qualitatively new features and can, in particular, include the double porosity model. This model was investigated in [11] with formal calculations and with mathematical rigour in [10] and [28]. A stochastic setting has been studied in [9] in the nondegenerate case and the problem with hysteresis has been studied in [6], again in the nondegenerate case.

A consequence of a degenerate ellipticity coefficient is that no uniform bounds for the pressure gradients in $L^{2}$-spaces are available. This problem is often treated with the help of the global pressure function, a tool of limited use in a setting with internal interfaces. As a consequence, the above quoted homogenization results are restricted to cases in which some smallness assumption is satisfied. In [8], this is an explicit smallness assumption concerning the jump of the global pressure, see their inequality (4.20). In [10], the smallness is imposed by using a permeability of order $\varepsilon^{2}$ in one of two media. We want to emphasize that this assumption is physically justified in many situations. In [28], more general permeabilities in the second medium are 
studied. The author uses the scaling $\varepsilon^{\varpi}$ and treats all cases with $\varpi>0$. In short, we may say that our works extends [28] to the case $\varpi=0$.

Finally, we mention [5] for a homogenization study in the compressible case. In that contribution, no $y$-dependence of the coefficients $\lambda_{w}(s)$ or $\rho_{g}(p)$ is permitted.

\section{Problem description and solution concepts}

\subsection{Domain and boundary conditions}

Let $\Omega \subset \mathbb{R}^{n}$ be bounded with Lipschitz boundary. Let $\Sigma_{j} \subset \partial \Omega$ be Dirichlet boundaries with positive $n-1$-dimensional measure, let $\Gamma_{j} \subset \partial \Omega$ be Neumann boundaries such that $\Sigma_{j} \cap \Gamma_{j}=\emptyset, \bar{\Sigma}_{j} \cup \bar{\Gamma}_{j}=\partial \Omega$. As boundary conditions we impose a no-flux condition for fluid $j$ on $\Gamma_{j}$, and the Dirichlet condition $p_{j}-U_{j}=0$ on $\Sigma_{j}$ for two given functions $U_{j} \in H^{1}(\Omega)$. We furthermore assume that initial values $S_{j} \in L^{2}(\Omega,[0,1])$ are given with $S_{w}+S_{n}=1$ and such that $\tilde{p}_{c}\left(S_{w}\right) \in L^{\infty}(\Omega)$.

\subsection{Assumptions on the coefficients}

Permeabilities. We assume that, for numbers $0<c_{0}<C_{0}<\infty$, and $j=w, n$, there holds, independent of $x$ and $y$,

$$
\begin{aligned}
& c_{0} \leq K_{j} \leq C_{0}, \quad k_{j} \leq C_{0}, \quad k_{n} \geq c_{0}, \\
& k_{w}(0)=0, \quad k_{w}(s)>0 \text { strictly monotone with } \\
& 0 \leq \partial_{s} k_{w}(s) \leq C_{0} \sqrt{k_{w}(s)} \quad \forall s \in(0,1] .
\end{aligned}
$$

The strict positivity assumption $k_{n} \geq c_{0}$ on the non-wetting permeability allows us to concentrate here on the degeneracy in $s=0$, which simplifies notation.

We will derive the two-scale limit system first in the non-degenerate case. In this case we assume that also the second relative permeability is bounded from below,

$$
k_{w} \geq c_{0}
$$

Capillary pressure. We assume that, for $0<c_{1}$, there holds, for every index $m$

$$
\begin{array}{ll}
\tilde{p}_{c}^{m} \in C^{1}([0,1), \mathbb{R}), & p_{c}^{m}(s)= \begin{cases}\left\{\tilde{p}_{c}^{m}(s)\right\} & \text { for } s>0 \\
\left(-\infty, \tilde{p}_{c}^{m}(0)\right] & \text { for } s=0,\end{cases} \\
c_{1} \leq \partial_{s} \tilde{p}_{c}^{m} \text { on }[0,1), & \tilde{p}_{c}^{m}(s) \rightarrow \infty \text { for } s \rightarrow 1 .
\end{array}
$$

We remark that the second assumption in (2.6) introduces a slightly asymmetric behavior, but our assumption is in accordance with experimental data. It simplifies the notation in the following since it helps to concentrate the analysis to the degeneracy at the point $s=s_{w}=0$.

We furthermore introduce a mild regularity assumption on the product $\Psi=k \cdot p_{c}$ as follows. For every $m \leq M$ and $y \in Y_{m}$, after an extension to boundary points, the 

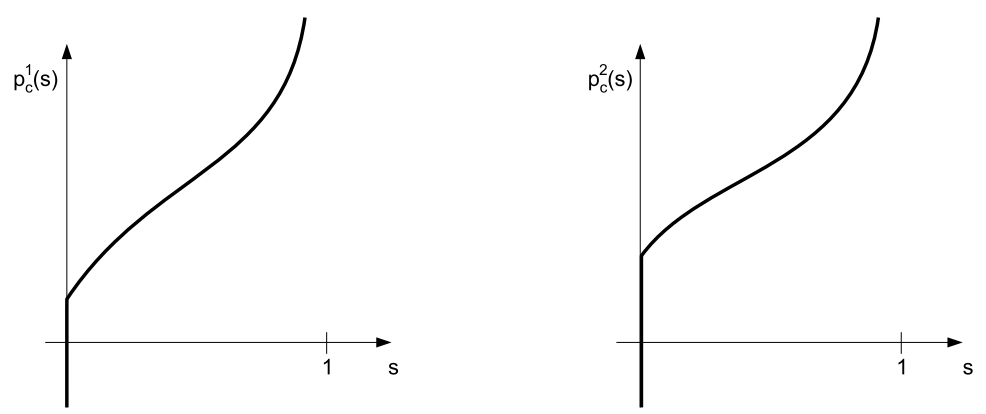

Figure 1: Sketch of two strictly monotone and multi-valued $p_{c}$-curves. The curve on the left is $p_{c}^{1}(s)$ for subdomain $m=1$ and on the right is $p_{c}^{2}(s)$ for subdomain $m=2$. The entry pressures are ordered such that $\max p_{c}^{2}(0)>\max p_{c}^{1}(0)$, the multivalued functions satisfy $p_{c}^{m}(0)=\left(-\infty, \max p_{c}^{m}(0)\right]$. The transformation map $\tilde{\Phi}_{2,1}=$ $\left(p_{c}^{2}\right)^{-1} \circ p_{c}^{1}$ is continuous on $[0,1]$.

maps

$$
\begin{aligned}
& \Psi(x, y, .):\left[0, k_{w}(x, y, 1)\right) \rightarrow \mathbb{R}, \quad \kappa \mapsto \kappa \cdot \tilde{p}_{c}\left(k_{w}^{-1}(\kappa)\right) \\
& \Psi, \frac{\partial}{\partial \kappa} \Psi: \bigcup_{(x, y) \in \bar{\Omega} \times \bar{Y}_{m}}(x, y) \times\left[0, k_{w}(x, y, 1)\right) \rightarrow \mathbb{R} \quad \text { are continuous. }
\end{aligned}
$$

Under our other assumptions, (2.7) is satisfied e.g. for polynomials $k_{w}$. Indeed, let us assume that $\partial_{s}\left[\log \left(k_{w}(s)\right)\right]$ extends continuously to $s=0$ with positive values. Then (2.7) follows from $\partial_{\kappa} \Psi(\kappa)=\tilde{p}_{c}(s)+\kappa \partial_{s} \tilde{p}_{c}(s)\left(\partial_{s} k_{w}(s)\right)^{-1}$ for $s \in(0,1)$ and $\kappa=k_{w}(s)$.

Interface condition maps. In order to encode interface conditions, we additionally introduce transformation functions as follows. By relabeling if necessary, we may assume in the following without loss of generality that the entry pressures $\max p_{c}^{m}(0)$ are ordered, $\max \left(p_{c}^{m}(0)\right) \leq \max \left(p_{c}^{m^{\prime}}(0)\right)$ for every pair $1 \leq m, m^{\prime} \leq M$ with $m^{\prime}<m$, the argument of the maximum is an interval of the form $(-\infty, \rho]$ (cf. Figure 1). For all pairs $m^{\prime}<m$ we introduce the transformation maps

$$
\begin{aligned}
& \tilde{\Phi}_{m, m^{\prime}}(x, .):=\left(p_{c}^{m}(x, .)\right)^{-1} \circ p_{c}^{m^{\prime}}(x, .):[0,1] \rightarrow[0,1], \\
& \Phi_{m, m^{\prime}}(x, .):=k_{w}^{m}(x, .) \circ \tilde{\Phi}_{m, m^{\prime}}(x, .) \circ\left(k_{w}^{m^{\prime}}(x, .)\right)^{-1} .
\end{aligned}
$$

The maps are defined such that the following holds. If $x$ is a point on $\partial \Omega_{m}^{\varepsilon} \cap \partial \Omega_{m^{\prime}}^{\varepsilon}$ and both pressure functions $p_{w}$ and $p_{n}$ are continuous, then the two traces $s_{w}^{m}(x)$ and $s_{w}^{m^{\prime}}(x)$ of the wetting saturation satisfy $s_{w}^{m}(x)=\tilde{\Phi}_{m, m^{\prime}}\left(x, s_{w}^{m^{\prime}}(x)\right)$. The other transformation map $\Phi_{m, m^{\prime}}$ maps the permeability value of one side to the corresponding permeability value of the other side. We assume that, for all $m^{\prime}<m$,

$$
\Phi_{m, m^{\prime}}, \frac{\partial}{\partial \kappa} \Phi_{m, m^{\prime}}: \bigcup_{(x, y) \in \bar{\Omega} \times \bar{Y}_{m}}(x, y) \times\left[0, k_{w}(x, y, 1)\right] \rightarrow \mathbb{R} \quad \text { are continuous. }
$$




\subsection{Solutions in heterogeneous media}

Our aim is to study solutions of the two-phase flow system (1.1)-(1.3) for the oscillatory coefficients of (1.4)-(1.6). For a given length scale $\varepsilon>0$ we hence study the following system of equations.

$$
\begin{aligned}
\partial_{t} s_{j}^{\varepsilon} & =\nabla \cdot q_{j}^{\varepsilon} & & \text { in } \Omega \text { for } j=w, n \\
q_{j}^{\varepsilon} & =K_{j}^{\varepsilon} k_{j}^{\varepsilon}\left(s_{j}^{\varepsilon}\right) \nabla p_{j}^{\varepsilon} & & \text { in } \Omega \text { for } j=w, n \\
s_{w}^{\varepsilon}+s_{n}^{\varepsilon} & =1, \quad p_{w}^{\varepsilon}-p_{n}^{\varepsilon} \in p_{c}^{\varepsilon}\left(s_{w}^{\varepsilon}\right) & & \text { in } \Omega,
\end{aligned}
$$

where we omitted once more the dependence on $t \in[0, T)$. We start our analysis by giving a precise description of our solution concept in the non-degenerate case.

Definition 2.1 (Strong solutions). We say that the six functions $\left(s_{j}^{\varepsilon}, p_{j}^{\varepsilon}, q_{j}^{\varepsilon}\right)$ are a strong solution to system (2.11)-(2.13) if the following holds. The functions are of the classes

$$
s_{j}^{\varepsilon} \in L^{\infty}(\Omega \times(0, T) ;[0,1]), \quad q_{j}^{\varepsilon} \in L^{2}\left(\Omega \times(0, T) ; \mathbb{R}^{n}\right), \quad p_{j}^{\varepsilon} \in L^{2}\left(0, T ; H^{1}(\Omega)\right),
$$

relation (2.11) holds in the distributional sense and both relations (2.12) and (2.13) hold pointwise almost everywhere.

The above choice of function spaces already encodes interface conditions at internal boundaries. Relation (2.11) contains, in a weak form, a continuity condition for the fluxes, the $H^{1}(\Omega)$ condition for pressures encodes, in a weak form, a continuity of the pressures across interfaces.

Proposition 2.1 (Existence of strong solutions in the non-degenerate case). Let the assumptions (2.1)-(2.6) on the coefficients be satisfied, in particular, we assume with (2.4) the non-degeneracy of the permeability of the wetting fluid. For a domain, initial and boundary data as described above, there exists a strong solution to the two-phase flow system (2.11)-(2.13). With a constant $C$ which depends only on the initial and boundary data, there holds an estimate

$$
\sum_{j \in\{w, n\}} \int_{0}^{T} \int_{\Omega} k_{j}^{\varepsilon}\left(s_{j}^{\varepsilon}\right)\left|\nabla p_{j}^{\varepsilon}\right|^{2} \leq C .
$$

Proof. We derive the a priori estimate (2.15) in section 4.1, see inequality (4.2). The inequality implies, in the non-degenerate case, an estimate for both pressure functions, $p_{j}^{\varepsilon} \in L^{2}\left(0, T ; H^{1}(\Omega)\right)$.

The existence result is classical, see $[13,20]$. It can be derived, e.g., with a discretization scheme exploiting an a priori estimate of the form (2.15) for the approximate solutions.

Definition 2.2 (Weighted solutions). We say that the six functions $\left(s_{j}^{\varepsilon}, q_{j}^{\varepsilon}, p_{j}^{\varepsilon}\right)$ provide $a$ weighted solution to system (2.11)-(2.13) if the following holds. The capillary 
pressure inclusion holds as an equality, $p_{w}^{\varepsilon}-p_{n}^{\varepsilon}=\tilde{p}_{c}^{\varepsilon}\left(s_{w}^{\varepsilon}\right)$ and $s_{w}^{\varepsilon}+s_{n}^{\varepsilon}=1$ hold almost everywhere, on the $M$ open disjoint subdomains $\Omega_{m}^{\varepsilon}$ the functions are of the class

$$
\begin{aligned}
& s_{j}^{\varepsilon} \in L^{\infty}(\Omega \times(0, T) ;[0,1]), \quad q_{j}^{\varepsilon} \in L^{2}\left(\Omega \times(0, T) ; \mathbb{R}^{n}\right), \\
& p_{n}^{\varepsilon} \in L^{2}\left(0, T ; H^{1}(\Omega)\right), \quad p_{w}^{\varepsilon} \in L^{2}(\Omega \times(0, T) ; \mathbb{R}), \\
& \left.k_{w}^{\varepsilon}\left(s_{w}^{\varepsilon}\right)\right|_{\Omega_{m}^{\varepsilon}}, \quad \psi_{w}^{\varepsilon}:=\left.k_{w}^{\varepsilon}\left(s_{w}^{\varepsilon}\right) p_{w}^{\varepsilon}\right|_{\Omega_{m}^{\varepsilon}} \in L^{2}\left(0, T ; H^{1}\left(\Omega_{m}^{\varepsilon}\right)\right),
\end{aligned}
$$

the conservation law (2.11) holds in the distributional sense, the negative flux relation (2.12) for $j=n$ holds almost everywhere. Instead of (2.12) for $j=w$ we demand that

$$
q_{w}^{\varepsilon}=K_{w}^{\varepsilon}\left(\nabla\left[k_{w}^{\varepsilon}\left(s_{w}^{\varepsilon}\right) p_{w}^{\varepsilon}\right]-\nabla\left[k_{w}^{\varepsilon}\left(s_{w}^{\varepsilon}\right)\right] p_{w}^{\varepsilon}\right)
$$

holds in each open subdomain $\Omega_{m}^{\varepsilon}$. In order to encode continuity of the pressure $p_{w}$ across interfaces, we additionally demand

$$
\operatorname{trace}_{m}\left(k_{w}^{\varepsilon}\left(s_{w}^{\varepsilon}\right)\right)=\Phi_{m, m^{\prime}}\left(x, \operatorname{trace}_{m^{\prime}}\left(k_{w}^{\varepsilon}\left(s_{w}^{\varepsilon}\right)\right)\right)
$$

on $\partial \Omega_{m^{\prime}}^{\varepsilon} \cap \partial \Omega_{m}^{\varepsilon}$ with $m^{\prime}<m$. Here trace ${ }_{m}$ denotes the trace from the interior of $\Omega_{m}^{\varepsilon}$.

We note that the regularity assumption (2.18) on $k_{w}^{\varepsilon}\left(s_{w}^{\varepsilon}\right)$ allows to evaluate the traces in (2.20). Furthermore we observe that in (2.19) the right hand side vanishes almost everywhere in the set $\left\{(x, t): k_{w}^{\varepsilon}\left(s_{w}^{\varepsilon}(x, t)\right)=0\right\}$. This follows from Stampacchia's lemma since both functions $k_{w}^{\varepsilon} p_{w}^{\varepsilon}$ and $k_{w}^{\varepsilon}$ of which gradients are evaluated, vanish on this set. In this sense, in regions with a vanishing wetting saturation, we impose a vanishing flux, but we do not select a value for the wetting pressure.

Proposition 2.2 (Existence of weighted solutions in the degenerate case). Let assumptions (2.1)-(2.3) and (2.5)-(2.10), but not necessarily (2.4). For a sequence $\delta \searrow 0$ let $k_{w}^{\delta} \rightarrow k_{w}$ be an approximation of the permeability in $C^{0}([0,1], \mathbb{R})$ such that (2.1)-(2.6) hold for $k_{w}^{\delta}$ and with $k_{w}^{\delta} \geq \delta$. Let $s_{j}^{\delta}, q_{j}^{\delta}$, and $p_{j}^{\delta}$ be solutions to the corresponding non-degenerate problem. We assume that a uniform bound $\left\|p_{j}^{\delta}\right\|_{L^{\infty}\left(\Omega_{T}\right)} \leq C$ is satisfied for both pressure functions.

Under these conditions, there exists a subsequence $\delta \rightarrow 0$ and limit functions $s_{j}$, $q_{j}$, and $p_{j}$ which are a weighted solution to the degenerate two-phase flow system (2.11)-(2.13) with coefficient $k_{w}$.

Since our interest here regards the homogenization procedure, we do not investigate conditions that guarantee the boundedness of the pressures. From a physical point of view, for bounded pressure values along the boundaries, one will always expect a boundedness of the pressures in the interior. Regarding mathematical proofs of the boundedness assumption we mention that in [21] uniform bounds are derived with the help of maximum principles.

Proof. We omit the index $\varepsilon$ which stands for the small scale size. Instead, the solutions carry an index $\delta$ which stands for the non-degenerate approximation.

Solutions to the non-degenerate two-phase flow system (2.11)-(2.13) satisfy the pressure bounds of (2.15) as noted before in Proposition 2.1. Additionally, under the assumption of uniform $L^{\infty}$-bounds on the pressure function, with a constant $C$ 
depending only on initial and boundary data and this uniform bound, there holds an estimate

$$
\int_{0}^{T} \int_{\Omega_{m}^{\varepsilon}}\left|\nabla\left(k_{w}^{\delta}\left(s_{w}^{\delta}\right)\right)\right|^{2}+\int_{0}^{T} \int_{\Omega_{m}^{\varepsilon}}\left|\nabla \psi_{w}^{\delta}\right|^{2} \leq C .
$$

for every $m \leq M$. This estimate is shown in section 4.2 , see inequality (4.3).

We can therefore choose a subsequence and limit functions such that, in the limit $\delta \rightarrow 0$

$$
\begin{aligned}
& p_{n}^{\delta} \rightarrow p_{n} \text { in } L^{2}\left(\Omega_{T}\right), \quad q_{j}^{\delta} \rightarrow q_{j} \text { in } L^{2}\left(\Omega_{T}\right), \\
& \psi_{w}^{\delta} \rightarrow \psi_{w}, k_{w}^{\delta} \rightarrow k_{w} \quad \text { in } H^{1}\left(\Omega_{T}^{m}\right), \quad s_{j}^{\delta} \rightarrow s_{j} \text { in } L^{2}\left(\Omega_{T}\right) .
\end{aligned}
$$

In this list, all convergences are a direct consequence of the a priori estimates, with the exception of the last convergence, which follows from the strong convergence of $k_{w}^{\delta}=k_{w}^{\delta}\left(s_{w}^{\delta}\right)$. In particular, the limit equations are in the appropriate function spaces as demanded in (2.16)-(2.18) and $s_{w}+s_{n}=1$ is satisfied.

We now define $p_{w}:=p_{n}+\tilde{p}_{c}\left(s_{w}\right)$. The strong limit $\psi_{w}$ satisfies $\psi_{w}=k_{w} p_{w}$ by the strong convergence of $k_{w}^{\delta}$. We exploit here that we can define the pressure value $p_{w}$ arbitrarily on the set where $k_{w}=\psi_{w}=0$ holds. On the remaining set we have the strong convergence $s_{j}^{\delta} \rightarrow s_{j}$ and hence $p_{w}^{\delta} \in p_{n}^{\delta}+p_{c}\left(s_{w}^{\delta}\right) \rightarrow p_{n}+\tilde{p}_{c}\left(s_{w}\right)=p_{w}$ pointwise almost everywhere.

The distributional relation (2.11) holds in the limit $\delta \rightarrow 0$ since derivatives commute with distributional limits. Furthermore, (2.12) for $j=n$ holds by the strong convergence of $k_{n}^{\delta}$. The interface condition (2.20) is satisfied for the approximate solutions $(\delta>0)$ and continues to hold in the limit $\delta \rightarrow 0$ due to the weak $H^{1}\left(\Omega_{T}^{m}\right)$ convergence of the permeabilities.

It remains to verify (2.19). We start from the strong relation (2.12) which we write with the product rule of $H^{1}$-functions as

$$
q_{w}^{\delta}=K_{w}\left(\nabla \psi_{w}^{\delta}-p_{w}^{\delta} \nabla k_{w}^{\delta}\right)
$$

The difficulty in this expression is that we have only the weak convergences for both $p_{w}^{\delta}$ and $\nabla k_{w}^{\delta}$. We can argue as follows. We multiply the relation with $k_{w}^{\delta}$, such that weak limits exist for both sides,

$$
k_{w} q_{w} \leftarrow k_{w}^{\delta} q_{w}^{\delta}=K_{w}\left(k_{w}^{\delta} \nabla \psi_{w}^{\delta}-\psi_{w}^{\delta} \nabla k_{w}^{\delta}\right) \rightarrow K_{w}\left(k_{w} \nabla \psi_{w}-\psi_{w} \nabla k_{w}\right)
$$

in $L^{2}\left(\Omega_{T}\right)$. On the set of points $(x, t)$ with $k_{w}(x, t)>0$, we divide by $k_{w}(x, t)$ and obtain the pointwise relation (2.19). For the remaining set with $k_{w}(x, t)>0$ we have already observed that the right hand side of (2.19) vanishes almost everywhere by the lemma of Stampacchia. The flux function is the weak limit of $q_{w}^{\delta}=k_{w}^{\delta} \nabla p_{w}^{\delta}=$ $\sqrt{k_{w}^{\delta}} \cdot \sqrt{k_{w}^{\delta}} \nabla p_{w}^{\delta}$ where the factor $\sqrt{k_{w}^{\delta}} \nabla p_{w}^{\delta}$ is bounded in $L^{2}\left(\Omega_{T}\right)$. This provides $q_{w}=0$ on the set with $k_{w}(x, t)=0$ and hence (2.19) also on this set.

\section{Main homogenization results}

We study a sequence $\varepsilon=\varepsilon_{i} \rightarrow 0$ and a corresponding family of solutions $\left(s_{j}^{\varepsilon}, p_{j}^{\varepsilon}, q_{j}^{\varepsilon}\right)$ to system $(2.11)-(2.13)$. In the case of a non-degenerate relative permeability $k_{w}$ we 
study a family of strong solutions and obtain for their two-scale limits the two-scale limiting problem (ND) described in Definition 3.1. In the case of a degenerate relative permeability $k_{w}$ we study weighted solutions and obtain the limiting system (D) described in Definition 3.2. The limiting problems (ND) and (D) coincide formally, but the formulation of problem (D) is taylored to the fact that the pressures need not have gradients of class $L^{2}$.

\subsection{The limit problem in the non-degenerate case}

Definition 3.1 (Strong limit problem in the non-degenerate case). We denote the following two-scale problem as problem (ND). We seek for eight functions $s_{j, 0}(x, y)$, $p_{j, 0}(x), p_{j, 1}(x, y), q_{j, 0}(x, y)$ in the function spaces

$$
\begin{aligned}
& p_{j, 0} \in L^{2}\left(0, T ; H^{1}(\Omega)\right), \quad s_{j, 0} \in L^{\infty}(\Omega \times Y \times(0, T) ;[0,1]), \\
& p_{j, 1} \in L^{2}\left((0, T) \times \Omega ; H_{\mathrm{per}}^{1}(Y)\right), \quad q_{j, 0} \in L^{2}\left(\Omega \times Y \times(0, T) ; \mathbb{R}^{n}\right) .
\end{aligned}
$$

The equations are

$$
\begin{aligned}
\partial_{t} f_{Y} s_{j, 0}(x, y) d y & =\nabla_{x} \cdot f_{Y} q_{j, 0}(x, y) d y \\
q_{j, 0}(x, y) & =K_{j}(x, y) k_{j}\left(x, y, s_{j, 0}(x, y)\right)\left(\nabla_{x} p_{j, 0}(x)+\nabla_{y} p_{j, 1}(x, y)\right), \\
\nabla_{y} \cdot q_{j, 0}(x, y) & =0 \\
s_{w, 0}(x, y)+s_{n, 0}(x, y) & =1, \\
p_{w, 0}(x)-p_{n, 0}(x) & \in p_{c}\left(x, y, s_{w, 0}(x, y)\right) .
\end{aligned}
$$

We demand (3.3) in the distributional sense on $\Omega \times(0, T)$ and the other relations pointwise almost everywhere.

Concerning the solvability of the limit problem (ND), we remark that the eight unknowns can, formally, be related to the eight equations. Relation (3.7) allows to reconstruct $s_{w, 0}(x, y)$ from the pressures $p_{j, 0}(x)$. In turn, relation (3.6) provides $s_{n, 0}(x, y)$. The negative fluxes $q_{j, 0}(x, y)$ are given by (3.4) and we regard (3.5) as an elliptic equations for $\left.p_{j, 1}(x, y)\right)$. With this reasoning, we have seen that (3.4)-(3.7) can determine all other quantities from the two macroscopic pressures $p_{j, 0}(x)$. If this is the case, the two macroscopic equations (3.3) have the structure

$$
\partial_{t} \mathcal{S}_{j}\left(p_{w, 0}(x), p_{n, 0}(x)\right)=\nabla_{x} \cdot \mathcal{F}_{j}\left(p_{w, 0}(x), p_{n, 0}(x), \nabla_{x} p_{w, 0}(x), \nabla_{x} p_{n, 0}(x)\right)
$$

for some functions $\mathcal{S}_{j}$ and $\mathcal{F}_{j}$. In the one-dimensional case, the above arguments can be made precise, see the effective one-dimensional system (5.7) in section 5 .

Theorem 1 (Non-degenerate homogenization). Let the domain $\Omega$, the time-interval $[0, T]$, the boundary conditions $U_{j}$ and the initial conditions $S_{j}$ be as described above. Let the coefficients satisfy assumptions (2.1)-(2.6), hence, in particular, the nondegeneracy condition (2.4). Let $p_{j}^{\varepsilon}, s_{j}^{\varepsilon}, q_{j}^{\varepsilon}$ be a family of strong solutions to the two-phase flow system (2.11)-(2.13) and let $p_{j, 0}, p_{j, 1}, s_{j, 0}$, and $q_{j, 0}$ be two-scale limit functions. Then the limit functions satisfy the two-scale limit system (ND) as described in Definition 3.1.

We note that the definition of the two-scale limit functions is made precise in (4.9)-(4.12). The theorem is shown in subsection 4.3. 


\subsection{The limit problem in the degenerate case}

Definition 3.2 (Weighted limit problem in the degenerate case). We denote the following two-scale problem as problem (D). The unknowns are seven functions $s_{j, 0}(x, y)$, $q_{j, 0}(x, y), p_{j, 0}(x), p_{n, 1}(x, y)$ as in the non-degenerate case, with function spaces as in (3.1)-(3.2). Two further unknowns are $\psi_{w, 1}(x, y)$ and $k_{w, 1}(x, y)$ in the space

$$
\left.\psi_{w, 1}\right|_{Y_{m}},\left.k_{w, 1}\right|_{Y_{m}} \in L^{2}\left((0, T) \times \Omega ; H^{1}\left(Y_{m}\right)\right) .
$$

We use $k_{j, 0}(x, y):=k_{j}\left(x, y, s_{j, 0}(x, y)\right)$ and $\psi_{w, 0}(x, y):=k_{w, 0}(x, y) p_{w, 0}(x)$ as abbreviations. The system replacing (3.3)-(3.7) is, for $j=w, n$,

$$
\begin{aligned}
\partial_{t} f_{Y} s_{j, 0}(x, y) d y & =\nabla_{x} \cdot f_{Y} q_{j, 0}(x, y) d y \\
q_{n, 0}(x, y) & =K_{n}(x, y) k_{n, 0}(x, y)\left(\nabla_{x} p_{n, 0}(x)+\nabla_{y} p_{n, 1}(x, y)\right), \\
q_{w, 0}(x, y) & =K_{w}(x, y)\left(\nabla_{x} \psi_{w, 0}(x)+\nabla_{y} \psi_{w, 1}(x, y)\right. \\
\nabla_{y} \cdot q_{j, 0}(x, y) & \left.=0, \quad-p_{w, 0}(x)\left[\nabla_{x} k_{w, 0}(x, y)+\nabla_{y} k_{w, 1}(x, y)\right]\right) \\
s_{w, 0}(x, y)+s_{n, 0}(x, y) & =1, \\
p_{w, 0}(x)-p_{n, 0}(x) & =\tilde{p}_{c}\left(x, y, s_{w, 0}(x, y)\right) .
\end{aligned}
$$

Since $\psi_{w, 1}$ may jump across material interfaces, equation (3.10) is understood as a distributional equation in each subdomain $Y_{m}$. To encode continuity of the wetting pressure in orders 0 and 1 we demand

$$
\begin{array}{ll}
\operatorname{trace}_{m}\left(k_{w, 0}\right)=\Phi_{m, m^{\prime}}\left(\operatorname{trace}_{m^{\prime}}\left(k_{w, 0}\right)\right) & \text { on } \partial Y_{m} \cap \partial Y_{m^{\prime}} \\
\operatorname{trace}_{m}\left(k_{w, 1}\right)=\frac{\partial}{\partial \kappa} \Phi_{m, m^{\prime}}\left(\operatorname{trace}_{m^{\prime}}\left(k_{w, 0}\right)\right) \cdot \operatorname{trace}_{m^{\prime}}\left(k_{w, 1}\right) & \text { on } \partial Y_{m} \cap \partial Y_{m^{\prime}}
\end{array}
$$

for every pair $1 \leq m^{\prime}<m \leq M$, traces are with respect to the variable $y$ in $Y_{m}$.

While $\psi_{w, 1}(x, y)$ is only a replacement of $p_{w, 1}(x, y)$, the additional scalar unknown $k_{w, 1}(x, y)$ must be determined from the additional equation

$$
\begin{aligned}
\psi_{w, 1}(x, y)=\frac{\partial}{\partial \kappa} \Psi( & \left.k_{w, 0}(x, y)\right) \cdot k_{w, 1}(x, y) \\
& +p_{n, 0}(x) k_{w, 1}(x, y)+k_{w, 0}(x, y) p_{n, 1}(x, y)
\end{aligned}
$$

where we used the function $\Psi$ of assumption (2.7).

We recall that the function $\Psi=\mathrm{id} \cdot\left(\tilde{p}_{c} \circ k_{w}^{-1}\right)$ of assumption (2.7) maps a permeability value $k_{w}$ to the corresponding capillary pressure contribution of $\psi_{w}$. Relation (3.16) should be regarded as the first order variant of the pressure condition (3.13). The functions $\psi_{w, 0}$ and $\psi_{w, 1}$ will appear as the two-scale limits

$$
\begin{aligned}
\psi_{w}^{\varepsilon}:=k_{w}^{\varepsilon} p_{w}^{\varepsilon} \stackrel{2}{\rightarrow} \psi_{w, 0}, & \psi_{w, 0}: \Omega \times Y \rightarrow \mathbb{R}, \\
\nabla \psi_{w}^{\varepsilon} \stackrel{2}{\rightarrow} \nabla_{x} \psi_{w, 0}+\nabla_{y} \psi_{w, 1}, & \psi_{w, 1}: \Omega \times Y \rightarrow \mathbb{R} .
\end{aligned}
$$


Theorem 2 (Homogenization in the degenerate case). Let the domain $\Omega$, the timeinterval $[0, T]$, the boundary conditions $U_{j}$ and the initial conditions $S_{j}$ be as described above with $\Omega_{\varepsilon}^{m}$ connected for all $m$. Let the coefficients satisfy assumptions (2.1)(2.3) and (2.5)-(2.10), but not necessarily (2.4). Let $s_{j}^{\varepsilon}, q_{j}^{\varepsilon}, p_{j}^{\varepsilon}$ be a family of weighted solutions of the two-phase flow system (2.11)-(2.13). We assume that the pressures $p_{j}^{\varepsilon}$ are uniformly bounded in $L^{\infty}$.

Let $s_{j, 0}, q_{j, 0}$, and $p_{j, 0}$ be two-scale limit functions in $L^{2}(\Omega \times Y \times(0, T))$. Then these limit functions satisfy, with an appropriate choice of three additional unknowns $p_{n, 1}(x, y), \psi_{w, 1}(x, y)$, and $k_{w, 1}(x, y)$, the two-scale limit system $(D)$ as described in Definition 3.2.

Theorem 2 on the degenerate homogenization result is shown in subsection 4.4.

We assume in the above theorem the connectedness of all subdomains $\Omega_{\varepsilon}^{m}$. This is done for ease of notation, it is possible to extend the result to the case when only one subdomain is connected, but in this case one has to assume that the connected domain is the best conducting medium. Conditions on the capillary pressures must insure in such a case that the control of the pressure in the connected subdomain provides a control for all pressure functions. Technically, our assumptions make the theorem applicable in the case of multiple subdomains only in three space dimensions.

\subsection{Formal equivalence of the solution concepts}

We want to include in this subsections two observations on the formal equivalence of different formulations of the limit system. Our first observation regards the comparison of the degenerate limit system (D) and the non-degenerate limit system (ND).

Lemma 3.1. We consider a solution to the degenerate homogenized problem (D) and assume the additional regularity property that $p_{w, 1}:=p_{n, 1}+\partial_{\kappa}\left(\tilde{p}_{c} \circ k_{w}^{-1}\right)\left(s_{w, 0}\right) k_{w, 1}$ is in the function space as given in (3.2). Then, the solution to the degenerate problem (D) provides also a solution to problem (ND).

Proof. We observe that the set of non-degenerate equations (3.3)-(3.7) coincides exactly with the set of degenerate equations (3.8)-(3.13), with the only exeption that (3.4) for $j=w$ is replaced by (3.10). We therefore only have to verify (3.4) for $q_{w, 0}(x, y)$.

With the function $p_{w, 1}:=p_{n, 1}+\partial_{\kappa}\left(\tilde{p}_{c} \circ k_{w}^{-1}\right)\left(s_{w, 0}\right) k_{w, 1}$ we calculate

$$
\begin{aligned}
\nabla_{y} \psi_{w, 1} & \stackrel{(3.16)}{=} \partial_{\kappa} \Psi\left(k_{w, 0}\right) \cdot \nabla_{y} k_{w, 1}+p_{n, 0} \nabla_{y} k_{w, 1}+k_{w, 0} \nabla_{y} p_{n, 1} \\
& =\left(\tilde{p}_{c}\left(s_{w, 0}\right)+k_{w, 0} \partial_{\kappa}\left(p_{c} \circ k_{w}^{-1}\right)\left(s_{w, 0}\right)\right) \cdot \nabla_{y} k_{w, 1}+p_{n, 0} \nabla_{y} k_{w, 1}+k_{w, 0} \nabla_{y} p_{n, 1} \\
& \stackrel{(3.13)}{=} p_{w, 0} \nabla_{y} k_{w, 1}+k_{w, 0}\left(\partial_{\kappa}\left(\tilde{p}_{c} \circ k_{w}^{-1}\right)\left(s_{w, 0}\right) \nabla_{y} k_{w, 1}+\nabla_{y} p_{n, 1}\right) \\
& =p_{w, 0} \nabla_{y} k_{w, 1}+k_{w, 0} \nabla_{y} p_{w, 1} .
\end{aligned}
$$

We can investigate the expression for $q_{w, 0}$ in (3.10). We use the product rule and insert the above calculation to obtain

$$
\begin{aligned}
& \nabla_{x} \psi_{w, 0}+\nabla_{y} \psi_{w, 1}-p_{w, 0}\left[\nabla_{x} k_{w, 0}+\nabla_{y} k_{w, 1}\right] \\
& =\nabla_{x} k_{w, 0} p_{w, 0}+k_{w, 0} \nabla_{x} p_{w, 0}+k_{w, 0} \nabla_{y} p_{w, 1}+\nabla_{y} k_{w, 1} p_{w, 0}-p_{w, 0}\left[\nabla_{x} k_{w, 0}+\nabla_{y} k_{w, 1}\right] \\
& =k_{w, 0}\left[\nabla_{x} p_{w, 0}+\nabla_{y} p_{w, 1}\right]
\end{aligned}
$$


which confirms (3.4). We emphasize that the calculation is done for each subdomain $Y_{m}$ separately, in the subdomains vanishes the gradient $\nabla_{y} k_{j, 0}(x, y)$ identically because of our structural assumptions in (1.5)-(1.6).

In the non-degenerate case, the equation (3.16) could be written in the following more intuitive and more symmetric form, using four new auxiliary variables $p_{w, 1}(x, y)$, $k_{n, 1}(x, y)$ and $s_{j, 1}(x, y)$. We emphasize that, due to the degeneracy of the problem, the meaning of these auxiliary variables cannot necessarily be made precise and system (3.19)-(3.22) has only formal character.

$$
\begin{aligned}
k_{j, 1}(x, y) & =\partial_{s} k_{j}\left(s_{j, 0}(x, y)\right) s_{j, 1}(x, y) \quad \text { for } j=w, n, \\
\psi_{w, 1}(x, y) & =p_{w, 0}(x) k_{w, 1}(x, y)+k_{w, 0}(x, y) p_{w, 1}(x, y) \\
s_{w, 1}(x, y)+s_{n, 1}(x, y) & =0 \\
p_{w, 1}(x, y)-p_{n, 1}(x, y) & =\partial_{s} \tilde{p}_{c}\left(x, y, s_{w, 0}(x, y)\right) s_{w, 1}(x, y) .
\end{aligned}
$$

Our next observation regards the formal equivalence of the degenerate limit system (D) with the symmetric formulation of (3.19)-(3.22).

Lemma 3.2. Let the four functions $k_{j}, \partial_{s} k_{j}, \partial_{s} p_{c}:[0,1] \rightarrow \mathbb{R}$ be continuous and positive and let $s_{w, 0}$ be bounded from below by a positive number. Then equation (3.16) is equivalent to the five equations (3.19)-(3.22), which include the four auxiliary variables $s_{j, 1}, k_{n, 1}$, and $p_{w, 1}$.

Proof. Let $k_{j, 1}, p_{j, 1}$ and $s_{j, 1}$ be a solution of system (3.19)-(3.22). We evaluate with $k=k_{w}(s)$ the derivative of $\Psi$ as $\partial_{\kappa} \Psi(k)=\tilde{p}_{c}(s)+k_{w} \partial_{s} \tilde{p}_{c}(s)\left(\partial_{s} k_{w}(s)\right)^{-1}$ and calculate for the right hand side of (3.16)

$$
\begin{aligned}
\partial_{\kappa} \Psi & \left(k_{w, 0}\right) \cdot k_{w, 1}+p_{n, 0} k_{w, 1}+k_{w, 0} p_{n, 1} \\
& =\left(\tilde{p}_{c}\left(s_{w, 0}\right)+k_{w, 0} \partial_{s} \tilde{p}_{c}\left(s_{w, 0}\right)\left(\partial_{s} k_{w}\left(s_{w, 0}\right)\right)^{-1}\right) \cdot k_{w, 1}+p_{n, 0} k_{w, 1}+k_{w, 0} p_{n, 1} \\
& \stackrel{(3.19)}{=} p_{w, 0} k_{w, 1}+k_{w, 0} \partial_{s} \tilde{p}_{c}\left(s_{w, 0}\right) s_{w, 1}+k_{w, 0} p_{n, 1} \\
& \stackrel{(3.22)}{=} p_{w, 0} k_{w, 1}+k_{w, 0}\left(p_{w, 1}-p_{n, 1}\right)+k_{w, 0} p_{n, 1} \\
& =p_{w, 0} k_{w, 1}+k_{w, 0} p_{w, 1} \stackrel{(3.20)}{=} \psi_{w, 1} .
\end{aligned}
$$

We have thus obtained (3.16). We emphasize that we have used also the macrorelation (3.13).

For the other implication we start with a solution of (D), in particular, $k_{w, 1}$ satisfies (3.16). We define $s_{w, 1}$ with (3.19) for $j=w$, then $s_{n, 1}$ with (3.21), $k_{n, 1}$ with (3.19) for $j=n$, and finally $p_{w, 1}$ with (3.22). It remains to check for these quantities that relation (3.20) is satisfied. This follows with the same calculation as above.

\section{Proofs}

\subsection{Energy estimates for the pressure}

Energy estimates are obtained by using $p_{j}^{\varepsilon}-U_{j}$ as a test-function in (2.11), where $U_{j}$ is the function that provides the boundary data. We add the equations for $j=w$ 
and $j=n$. This provides

$$
\begin{aligned}
& \sum_{j \in\{w, n\}} \int_{\Omega} p_{j}^{\varepsilon} \partial_{t} s_{j}^{\varepsilon}+\sum_{j \in\{w, n\}} \int_{\Omega} \nabla p_{j}^{\varepsilon} \cdot K_{j}^{\varepsilon} k_{j}^{\varepsilon}\left(s_{j}^{\varepsilon}\right) \nabla p_{j}^{\varepsilon} \\
& =\sum_{j \in\{w, n\}} \int_{\Omega} \nabla p_{j}^{\varepsilon} \cdot K_{j}^{\varepsilon} k_{j}^{\varepsilon}\left(s_{j}^{\varepsilon}\right) \nabla U_{j}+\sum_{j \in\{w, n\}} \int_{\Omega} \partial_{t} s_{j}^{\varepsilon} U_{j} .
\end{aligned}
$$

On the right hand side we use the Cauchy-Schwarz inequality, exploiting $U_{j} \in H^{1}(\Omega)$ and the boundedness $K_{j}, k_{j} \leq C_{0}$ to find

$$
\left|\int_{\Omega} \nabla p_{j}^{\varepsilon} \cdot K_{j}^{\varepsilon} k_{j}^{\varepsilon}\left(s_{j}^{\varepsilon}\right) \nabla U_{j}\right| \leq C\left(\int_{\Omega} K_{j}^{\varepsilon} k_{j}^{\varepsilon}\left(s_{j}^{\varepsilon}\right)\left|\nabla p_{j}^{\varepsilon}\right|^{2}\right)^{1 / 2} .
$$

With the Young inequality, this term can be absorbed into the second sum of the left hand side. For the further treatment of the first sum on the left hand side of (4.1), we use the algebraic relations of (2.13). The saturation relation $s_{n}^{\varepsilon}+s_{w}^{\varepsilon}=1$ provides $\partial_{t} s_{n}^{\varepsilon}=-\partial_{t} s_{w}^{\varepsilon}$. Exploiting additionally the capillary pressure relation, we find

$$
\sum_{j \in\{w, n\}} p_{j}^{\varepsilon} \partial_{t} s_{j}^{\varepsilon}=\left(p_{w}^{\varepsilon}-p_{n}^{\varepsilon}\right) \partial_{t} s_{w}^{\varepsilon}=p_{c}^{\varepsilon}\left(s_{w}^{\varepsilon}\right) \partial_{t} s_{w}^{\varepsilon}=\frac{d}{d t}\left[P_{c}^{\varepsilon}\left(s_{w}^{\varepsilon}\right)\right]
$$

where we used the family of primitive functions $P_{c}^{\varepsilon}$ with $\partial_{s} P_{c}^{\varepsilon}(x, s)=p_{c}^{\varepsilon}(x, s)$. The monotonicity and boundedness from below of $p_{c}^{\varepsilon}$ in $s$ implies that the primitives $P_{c}^{\varepsilon}$ with the normalization $P_{c}^{\varepsilon}(s=0)=0$ are all bounded from below. Together with our assumption on the initial values $S_{j}$, we obtain the boundedness of the first sum on the left hand side of (4.1). Since the functions $K_{j}^{\varepsilon}$ were assumed to be strictly positive, we obtain the a priori estimate

$$
\sum_{j \in\{w, n\}} \int_{0}^{T} \int_{\Omega} k_{j}^{\varepsilon}\left(s_{j}^{\varepsilon}\right)\left|\nabla p_{j}^{\varepsilon}\right|^{2} \leq C
$$

with $C$ depending on initial and boundary values, but not on $\varepsilon$.

\subsection{A priori estimates for $k_{w}$ and $\psi_{w}$.}

The fundamental observation for the definition of weighted solution is the following $H^{1}$-bound for the two quantities $k_{w}^{\varepsilon}:=k_{w}^{\varepsilon}\left(s_{w}^{\varepsilon}\right)$ and $\psi_{w}^{\varepsilon}:=k_{w}^{\varepsilon} p_{w}^{\varepsilon}$,

$$
\int_{0}^{T} \int_{\Omega_{m}^{\varepsilon}}\left|\nabla k_{w}^{\varepsilon}\right|^{2}+\int_{0}^{T} \int_{\Omega_{m}^{\varepsilon}}\left|\nabla \psi_{w}^{\varepsilon}\right|^{2} \leq C .
$$

In order to obtain (4.3), we first write $s_{w}^{\varepsilon}$ with the help of (2.13) as $s_{w}^{\varepsilon}=\left(p_{c}^{\varepsilon}\right)^{-1}\left(p_{w}^{\varepsilon}-\right.$ $\left.p_{n}^{\varepsilon}\right)$, such that the gradient satisfies, due to the strict monotonicity assumption (2.6),

$$
\left|\nabla s_{w}^{\varepsilon}\right|^{2}=\left|\frac{1}{\partial_{s} p_{c}^{\varepsilon}\left(s_{w}^{\varepsilon}\right)}\left(\nabla p_{w}^{\varepsilon}-\nabla p_{n}^{\varepsilon}\right)\right|^{2} \leq \frac{1}{c_{1}^{2}}\left|\nabla p_{w}^{\varepsilon}-\nabla p_{n}^{\varepsilon}\right|^{2} .
$$


This estimate allows to calculate with property (2.3)

$$
\begin{aligned}
\left|\nabla k_{w}^{\varepsilon}\right|^{2} & =\left|\partial_{s} k_{w}^{\varepsilon}\left(s_{w}^{\varepsilon}\right)\right|^{2}\left|\nabla s_{w}^{\varepsilon}\right|^{2} \leq C_{0}^{2} k_{w}^{\varepsilon}\left(s_{w}^{\varepsilon}\right)\left|\nabla s_{w}^{\varepsilon}\right|^{2} \\
& \leq\left(C_{0} / c_{1}\right)^{2} k_{w}^{\varepsilon}\left(s_{w}^{\varepsilon}\right)\left|\nabla p_{w}^{\varepsilon}-\nabla p_{n}^{\varepsilon}\right|^{2} \leq 2\left(C_{0} / c_{1}\right)^{2} k_{w}^{\varepsilon}\left(s_{w}^{\varepsilon}\right)\left(\left|\nabla p_{w}^{\varepsilon}\right|^{2}+\left|\nabla p_{n}^{\varepsilon}\right|^{2}\right) .
\end{aligned}
$$

The non-degeneracy of the non-wetting permeability in (2.1) and the boundedness of the wetting permeability allow to conclude from estimate (4.2) the boundedness of integrals over $k_{w}^{\varepsilon}\left(s_{w}^{\varepsilon}\right)\left|\nabla p_{n}^{\varepsilon}\right|^{2}$. Therefore, estimate (4.2) implies the a priori bound

$$
\int_{0}^{T} \int_{\Omega}\left|\nabla k_{w}^{\varepsilon}\right|^{2} \leq C
$$

Based on this observation, we can now calculate for $\psi_{w}^{\varepsilon}=k_{w}^{\varepsilon} p_{w}^{\varepsilon}$ in the case that $p_{w}^{\varepsilon}$ is uniformly bounded

$$
\int_{0}^{T} \int_{\Omega}\left|\nabla \psi_{w}^{\varepsilon}\right|^{2} \leq C \int_{0}^{T} \int_{\Omega}\left(\left|\nabla k_{w}^{\varepsilon}\right|^{2}+k_{w}^{\varepsilon}\left|\nabla p_{w}^{\varepsilon}\right|^{2}\right) \leq C
$$

by (4.2) and (4.4).

\subsection{Two-scale limits and proof in the non-degenerate case}

We use two-scale convergence as described in [1]; the (weak) two-scale convergence $u^{\varepsilon} \stackrel{2}{\rightarrow} u$ allows to extract $\varepsilon Y$-periodic behavior of the sequence of functions $u^{\varepsilon}=u^{\varepsilon}(x)$ and encodes it in the function $u=u(x, y)$ with $y \in Y$. We furthermore use strong two-scale convergence as was investigated e.g. in [22, 29].

Definition 4.1. We say that a sequence $u^{\varepsilon} \in L^{2}(\Omega)$ is strongly two-scale convergent to $u \in L^{2}(\Omega \times Y)$, iff

$$
u^{\varepsilon} \stackrel{2}{\rightarrow} u \quad \text { and } \quad\left\|u^{\varepsilon}\right\|_{L^{2}(\Omega)} \rightarrow\|u\|_{L^{2}(\Omega \times Y)} .
$$

We note that, just as in the case of two-scale convergence, the concept can be transferred directly to space-time domains $\Omega_{T}$, in which the fine-scale expansion is performed only in the spatial parameters $x \in \Omega$ and not in the time variable $t \in[0, T]$. Below, we always treat the time variable as a parameter and omit dependences on $t$.

An equivalent definition of strong two-scale convergence can be given as follows. A sequence $u^{\varepsilon} \stackrel{2}{\rightarrow} u$ is strongly two-scale convergent, if, for every (weakly) two-scale convergent sequence $v^{\varepsilon} \stackrel{2}{\rightarrow} v$, there holds

$$
\lim _{\varepsilon \rightarrow 0} \int_{\Omega} u^{\varepsilon}(x) v^{\varepsilon}(x) d x=\int_{\Omega} \int_{Y} u(x, y) v(x, y) d y d x .
$$

The full strength of strong two-scale convergence becomes clear with the following result. Let $u^{\varepsilon} \stackrel{2}{\rightarrow} u$ be a strongly two-scale convergent sequence. Then there holds

$$
\lim _{\varepsilon \rightarrow 0}\left\|u^{\varepsilon}(x)-u\left(x, \frac{x}{\varepsilon}\right)\right\|_{L^{2}(\Omega)}=0 .
$$


We note that, in the setting of [29], a Caratheodory property of the limit function $u$ is assumed in the derivation of (4.8). On the other hand, no such assumptions are necessary for the Lebesgue measure setting in [22]; nevertheless, the authors of the latter work emphasize that also the appropriate choice of a space of test-functions is important. Vice versa, property (4.8) also implies the strong two-scale convergence, hence (4.8) can be used as a characterization of strong two-scale convergence.

In the proofs of our main theorems below we will use the following facts.

Lemma 4.1 (Piecewise $H^{1}$-bounds, strong two-scale convergence, nonlinear functions). Let $Y_{m} \subset Y$ and $\Omega_{m}^{\varepsilon}=\Omega \cap \bigcup_{k} \varepsilon\left(k+Y_{m}\right)$ be subdomains such that every $\Omega_{m}^{\varepsilon}$ is a connected Lipschitz-subdomain of $\Omega$.

(i) Let $u^{\varepsilon}$ be a bounded sequence in $H^{1}\left(\Omega_{m}^{\varepsilon}\right)$, we identify $u^{\varepsilon}$ with its trivial extension setting $u^{\varepsilon}=0$ in $\Omega \backslash \Omega_{m}^{\varepsilon}$ such that we may also write $u^{\varepsilon} \in L^{2}(\Omega)$. Then, along a subsequence and for a limit function $u \in L^{2}\left(\Omega, H^{1}\left(Y_{m}\right)\right)$, the sequence $u^{\varepsilon}$ converges strongly in two scales to $u$.

(ii) Let the sequence $u^{\varepsilon} \stackrel{2}{\rightarrow} u$ be strongly two-scale convergent and let $q: \mathbb{R} \rightarrow \mathbb{R}$ be a Lipschitz continuous function. We consider $v^{\varepsilon}:=q \circ u^{\varepsilon} \in L^{2}(\Omega)$ and $v:=q \circ u \in$ $L^{2}(\Omega \times Y)$. Then there holds the strong two-scale convergence $v^{\varepsilon} \stackrel{2}{\rightarrow} v$.

(iii) Let the sequence $u^{\varepsilon}: \Omega \rightarrow[a, b]$ have values in the interval $[a, b]$. Let $q$ : $[a, b] \rightarrow \mathbb{R}$ be a continuous nonlinear function, continuously differentiable on $(a, b)$ with $q^{\prime}>0$ and $q^{\prime}$ bounded on every interval $[a+\delta, b]$ with $\delta>0$. Then the strong two-scale convergence $u^{\varepsilon} \stackrel{2}{\rightarrow} u$ implies the strong two-scale convergence $v^{\varepsilon} \rightarrow v$ for $v^{\varepsilon}=q \circ u^{\varepsilon}$ and $v=q \circ u$.

In item (iii) we think, e.g., of a square-root function, $q(\xi)=\sqrt{\xi}, q:[0,1] \rightarrow[0,1]$.

Sketch of proof for Lemma 4.1. Property (i) is a fact that is used in various forms for homogenization problems in domains with holes. Early contributions use extension operators and require regular subdomains. For the statement above we refer to the approach of [2].

Regarding (ii) it suffices to calculate with the Lipschitz constant $C_{q}$ of the function $q$ and with property (4.8)

$$
\int_{\Omega}\left|v^{\varepsilon}(x)-v\left(x, \frac{x}{\varepsilon}\right)\right|^{2} \leq C_{q}^{2} \int_{\Omega}\left|u^{\varepsilon}(x)-u\left(x, \frac{x}{\varepsilon}\right)\right|^{2} \rightarrow 0 .
$$

This shows property (4.8) for the sequence $v^{\varepsilon}$ and the function $v$ and thus the strong two-scale convergence of $v^{\varepsilon}$.

To check property (iii), we assume without loss of generality $a=0$. We introduce, for the arbitrarily small parameter $\delta>0$, the set of good points $G_{\varepsilon, \delta}=\{x \in \Omega$ : $u^{\varepsilon}(x) \leq 2 \delta$ and $\left.u(x, x / \varepsilon) \leq 2 \delta\right\}$; loosely speaking, both $u^{\varepsilon}$ and the limit function are small on this set. Secondly, we use the set of points $H_{\varepsilon, \delta}=\left\{x \in \Omega: u^{\varepsilon}(x) \geq\right.$ $\delta$ and $u(x, x / \varepsilon) \geq \delta\}$, where both functions $u^{\varepsilon}$ and $u$ are large. The two sets do not necessarily cover $\Omega$, since one function may be small while the other is large. We introduce the corresponding "bad" sets $B_{\varepsilon, \delta}^{1}:=\left\{x \in \Omega: u^{\varepsilon}(x) \geq 2 \delta\right.$ and $u(x, x / \varepsilon) \leq$ $\delta\}$ and $B_{\varepsilon, \delta}^{2}:=\left\{x \in \Omega: u^{\varepsilon}(x) \leq \delta\right.$ and $\left.u(x, x / \varepsilon) \geq 2 \delta\right\}$. Estimate (4.8) for strongly two-scale convergent sequences implies for the bad sets the smallness in measure, $\left|B_{\varepsilon, \delta}^{l}\right| \rightarrow 0$ for $\varepsilon \rightarrow 0$, any $\delta>0, l=1$ and $l=2$. 
For points $x \in G_{\varepsilon, \delta}$ we exploit the fact that both $v^{\varepsilon}=q\left(u^{\varepsilon}(x)\right)$ and $v(x, x / \varepsilon)=$ $q(u(x, x / \varepsilon))$ are close to $q(0)$. For points $x \in H_{\varepsilon, \delta}$ we use the argument of (ii), exploiting the Lipschitz continuity of $q:[a+\delta, b] \rightarrow \mathbb{R}$ with constant $C_{q, \delta}$. With the indicated decomposition of the domain we calculate

$$
\begin{aligned}
\int_{\Omega} \mid v^{\varepsilon}(x) & -\left.v\left(x, \frac{x}{\varepsilon}\right)\right|^{2} \leq 2 \int_{G_{\varepsilon, \delta}}\left(\left|v^{\varepsilon}(x)-q(0)\right|^{2}+|v(x, x / \varepsilon)-q(0)|^{2}\right) \\
& +C_{q, \delta}^{2} \int_{H_{\varepsilon, \delta}}\left|u^{\varepsilon}(x)-u\left(x, \frac{x}{\varepsilon}\right)\right|^{2}+C\left|B_{\varepsilon, \delta}^{1}\right|+C\left|B_{\varepsilon, \delta}^{2}\right| .
\end{aligned}
$$

The right hand side is arbitrarily small if we choose first $\delta>0$ small enough to obtain smallness of the first integral, and then $\varepsilon>0$ small in order to have smallness of the second integral and of the measures of the bad sets.

Due to the characterization in (4.8), this implies the strong two-scale convergence of the sequence $v^{\varepsilon}$.

Proof of Theorem 1. We study here the non-degenerate case, i.e. we assume that the positivity assumption $k_{j} \geq c_{0}$ of (2.4) is satisfied for both fluids $j=w$ and $j=n$. In this case, the a priori estimate (4.2) provides uniform estimates for both pressure functions $p_{j}^{\varepsilon} \in L^{2}\left(0, T ; H^{1}(\Omega)\right)$. Due to the strict monotonicity of $p_{c}$ in the variable $s$, these estimates carry over, in each subdomain $\Omega_{m}^{\varepsilon}$, to uniform estimates for $s_{w}^{\varepsilon}(x)=\left(p_{c}^{m}\right)^{-1}\left(p_{w}^{\varepsilon}(x)-p_{n}^{\varepsilon}(x)\right) \in H^{1}\left(\Omega_{m}^{\varepsilon}\right)$. We can therefore extract two-scale limits which read, omitting the time dependence in all expressions,

$$
\begin{aligned}
p_{j}^{\varepsilon} \stackrel{2}{\rightarrow} p_{j, 0}, & p_{j, 0}: \Omega \rightarrow \mathbb{R}, \\
\nabla p_{j}^{\varepsilon} \stackrel{2}{\rightarrow} \nabla_{x} p_{j, 0}+\nabla_{y} p_{j, 1}, & p_{j, 1}: \Omega \times Y \rightarrow \mathbb{R}, \\
s_{j}^{\varepsilon} \stackrel{2}{\rightarrow} s_{j, 0}, & s_{j, 0}: \Omega \times Y \rightarrow \mathbb{R}, \\
q_{j}^{\varepsilon} \stackrel{2}{\rightarrow} q_{j, 0}, & q_{j, 0}: \Omega \times Y \rightarrow \mathbb{R}^{n} .
\end{aligned}
$$

All two-scale convergences are with respect to the $L^{2}$-norm. Item (i) of Lemma 4.1 implies the strong two scale convergence in (4.9) and (4.11). In particular, we have now defined the eight variables of (3.1)-(3.2). It remains to verify the relations (3.3)-(3.7).

The weak limits of $s_{j}^{\varepsilon}$ and $q_{j}^{\varepsilon}$ are recovered as the averages of the two-scale limits,

$$
s_{j}^{\varepsilon} \rightarrow f_{Y} s_{j, 0}(x, y) d y, \quad q_{j}^{\varepsilon} \rightarrow f_{Y} q_{j, 0}(x, y) d y,
$$

hence (3.3) follows by taking the weak limit in (2.11).

In order to derive (3.4), we first observe that (2.12) implies

$$
\left(K_{j}^{\varepsilon} k_{j}^{\varepsilon}\left(s_{j}^{\varepsilon}\right)\right)^{-1} q_{j}^{\varepsilon}=\nabla p_{j}^{\varepsilon} \stackrel{2}{\rightarrow} \nabla_{x} p_{j, 0}+\nabla_{y} p_{j, 1} .
$$

On the left hand side, the factor $q_{j}^{\varepsilon}$ converges (weakly) in two-scales, see (4.12). In the other factor, we exploit the strong two scale convergence of $s_{j}^{\varepsilon}$, non-degeneracy of $k_{j}^{\varepsilon}$ and item (ii) of Lemma 4.1, to conclude the strong two scale convergence of

$$
\left(K_{j}^{\varepsilon} k_{j}^{\varepsilon}\left(s_{j}^{\varepsilon}\right)\right)^{-1} \stackrel{2}{\rightarrow}\left(K_{j}(x, y) k_{j}\left(x, y, s_{j, 0}(x, y)\right)\right)^{-1} .
$$


To be more precise, we use, in this conclusion, item (ii) of Lemma $4.1 M$ times, separately for each subdomain. With the help of property (4.7) of strong two-scale convergence one easily deduces the (weak) two-scale convergence of the product, $\left(K_{j}^{\varepsilon} k_{j}^{\varepsilon}\left(s_{j}^{\varepsilon}\right)\right)^{-1} q_{j}^{\varepsilon}$ on the left hand side of (4.13), converges in two scales to the function $\left(K_{j}(x, y) k_{j}\left(x, y, s_{j, 0}(x, y)\right)\right)^{-1} q_{j, 0}(x, y)$. This provides $(3.4)$.

We next want to verify (3.5), namely that the distribution $\nabla_{y} \cdot q_{j, 0}$ vanishes. By (2.11), for an arbitrary function $\phi \in C_{c}^{\infty}(\Omega \times Y \times(0, T), \mathbb{R})$ and the corresponding oscillatory function $\phi^{\varepsilon}(x, t)=\phi(x, x / \varepsilon, t)$ there holds

$$
\begin{aligned}
\left\langle\nabla_{y} \cdot q_{j, 0}\right\rangle(\phi) & =\int_{0}^{T} \int_{\Omega} \int_{Y} q_{j, 0}(x, y, t) \nabla_{y} \phi(x, y, t) \\
& =\lim _{\varepsilon \rightarrow 0} \int_{0}^{T} \int_{\Omega} q_{j}^{\varepsilon} \varepsilon \nabla \phi^{\varepsilon}=\lim _{\varepsilon \rightarrow 0} \int_{0}^{T} \int_{\Omega} s_{j}^{\varepsilon} \varepsilon \partial_{t} \phi^{\varepsilon}=0 .
\end{aligned}
$$

Relations (3.6) and (3.7) are immediate consequences of the algebraic conditions in (2.13). For the limit consideration leading to the nonlinear term in (3.7) we exploit, as in the derivation of (3.4), the strong two-scale convergence of $s_{w}^{\varepsilon}$ to $s_{w, 0}$.

This concludes the proof of Theorem 1 .

\subsection{Proof in the degenerate case}

Proof of Theorem 2. In the degenerate case we consider, in addition to the primal variables of saturations $s_{j}^{\varepsilon}$ and pressures $p_{j}^{\varepsilon}$, the wetting permeability $k_{w}^{\varepsilon}=k_{w}^{\varepsilon}\left(s_{w}^{\varepsilon}\right)$ and the weighted pressure $\psi_{w}^{\varepsilon}=k_{w}^{\varepsilon} p_{w}^{\varepsilon}$ as dependent variables.

Due to the $H^{1}$ estimates on subdomains, obtained in (4.3), we find two-scale limits

$$
\begin{aligned}
k_{w}^{\varepsilon} \stackrel{2}{\rightarrow} k_{w, 0}, & k_{w, 0}: \Omega \times Y \rightarrow \mathbb{R}, \\
\nabla k_{w}^{\varepsilon} \stackrel{2}{\rightarrow} \nabla_{x} k_{w, 0}+\nabla_{y} k_{w, 1}, \quad & k_{w, 1}: \Omega \times Y \rightarrow \mathbb{R},
\end{aligned}
$$

and analogous limits for $\psi_{w}^{\varepsilon}=k_{w}^{\varepsilon} p_{w}^{\varepsilon}$. Item (i) of Lemma 4.1 implies the strong two-scale convergence of $k_{w}^{\varepsilon}$ to $k_{w, 0}$ and of $\psi_{w}^{\varepsilon}$ to $\psi_{w, 0}$.

Step 1. Consequences of the strong two-scale convergence. The saturation variable $s_{w}^{\varepsilon}$ is related to the permeability through the algebraic relation $k_{w}^{\varepsilon}=k_{w}^{\varepsilon}\left(s_{w}^{\varepsilon}\right)$. Item (iii) of Lemma 4.1 is applicable and it implies the strong two-scale convergence $s_{w}^{\varepsilon} \stackrel{2}{\rightarrow}$ $s_{w, 0}$. Additionally, the lemma provides the characterization of the limit, $k_{w, 0}(x, y):=$ $k_{w}\left(x, y, s_{w, 0}(x, y)\right)$. Similarly, the strong two-scale convergence of $k_{w}^{\varepsilon}$ implies also relation $\psi_{w, 0}(x, y):=k_{w, 0}(x, y) p_{w, 0}(x, y)$ for the product term. The fact that $p_{w, 0}(x, y)$ is independent of $y$ in each subdomain $Y_{m}$ follows from the $y$-independence of $k_{w, 0}$ and $\psi_{w, 0}$ in each subdomain. Once the trace relation (3.14) is established, we conclude that $\tilde{p}_{c}\left(s_{w, 0}\right)$ is without jumps at interfaces and (3.13) for $p_{w, 0}(x, y)$ implies that the latter is indeed independent of $y \in Y$.

For the non-degenerate second phase, (4.2) provides the strong two-scale convergence of $p_{n}^{\varepsilon}$. With these first observations, we can now conclude the strong two-scale convergence of the wetting pressures $p_{w}^{\varepsilon}$. For this step to work, it is important to make a choice in the definition of the wetting pressure in the region with vanishing saturation. We made this choice by demanding the equality $p_{w}^{\varepsilon}-p_{n}^{\varepsilon}=\tilde{p}_{c}^{\varepsilon}\left(s_{w}^{\varepsilon}\right)$ in 
Definition 2.2 and, accordingly, the equality in (3.13). The properties of the function $\tilde{p}_{c}^{\varepsilon}$ allow to use item (iii) of Lemma 4.1 and to conclude from $p_{w}^{\varepsilon}-p_{n}^{\varepsilon}=\tilde{p}_{c}^{\varepsilon}\left(s_{w}^{\varepsilon}\right)$ the strong two-scale convergence $p_{w}^{\varepsilon} \stackrel{2}{\rightarrow} p_{w, 0}(x, y)=p_{n, 0}(x)+\tilde{p}_{c}\left(x, y, s_{w, 0}(x, y)\right)$, and, in particular, (3.13).

Step 2. Further limit equations. We have to verify the remaining equations of the system (3.8)-(3.16). Relations (3.8)-(3.12) with the exeption of (3.10) follow exactly as in the non-degenerate case, exploiting again the strong two-scale convergence of $s_{w}^{\varepsilon}$.

Relation (3.10) must be derived from the flux relation in the weighted solution setting, i.e. (2.19). This relation reads

$$
\left(K_{w}^{\varepsilon}\right)^{-1} q_{w}^{\varepsilon}=\nabla \psi_{w}^{\varepsilon}-\left(\nabla k_{w}^{\varepsilon}\right) p_{w}^{\varepsilon}
$$

The strong two-scale convergence of the pressure $p_{w}^{\varepsilon} \rightarrow p_{w, 0}(x, y)$ found in Step 1 allows to take the two-scale limit in the product term and we find (3.10).

The jump conditions (3.14) and (3.15) follow from (2.20), which provides

$$
\operatorname{trace}_{m}\left(k_{w}^{\varepsilon}\right)=\Phi_{m, m^{\prime}}\left(x, \operatorname{trace}_{m^{\prime}}\left(k_{w}^{\varepsilon}\right)\right) .
$$

The piecewise $H^{1}$-bound for $k_{w}^{\varepsilon}$ and the trace theorem allow to transfer this relation to the two-scale limit.

Finally, the relation between $\psi_{w, 1}$ and $k_{w, 1}$ of (3.16) follows from the definition of $\Psi(\kappa)=\kappa \tilde{p}_{c}\left(k_{w}^{-1}(\kappa)\right)$ in $(2.7)$, which allows to write

$$
\psi_{w}^{\varepsilon}=k_{w}^{\varepsilon} p_{w}^{\varepsilon}=\Psi\left(k_{w}^{\varepsilon}\right)+k_{w}^{\varepsilon} p_{n}^{\varepsilon},
$$

and hence also

$$
\nabla \psi_{w}^{\varepsilon}=\partial_{\kappa} \Psi\left(k_{w}^{\varepsilon}\right) \nabla k_{w}^{\varepsilon}+\nabla k_{w}^{\varepsilon} p_{n}^{\varepsilon}+k_{w}^{\varepsilon} \nabla p_{n}^{\varepsilon} .
$$

We take the two scale limit of both sides, exploiting the strong two-scale convergence of $k_{w}^{\varepsilon}$ and the pressure $p_{n}^{\varepsilon}$. A comparison with the $x$-gradient of the zero-order limit relation $\psi_{w, 0}(x, y)=k_{w, 0}(x, y) p_{w, 0}(x, y)=\Psi\left(k_{w, 0}(x, y)\right)+k_{w, 0}(x, y) p_{n, 0}(x)$ provides that the $y$-gradients of both sides in (3.16) coincide. Upon changing $\psi_{w, 0}(x, y)$ by an additive constant, we conclude (3.16). Hence, Theorem 2 is shown.

\section{Effective model in a one-dimensional case}

As an example for the non-degenerate limit problem of Definition 3.1, we study in this section the one-dimensional situation. We investigate a setting that had been treated before in $[12,15,16,26]$. Setting the space dimension to $n=1$, we use $Y=[-1,1)$ as a periodicity cell, set $K_{j}:=1$, and choose, for $x \in \Omega=(-L, L)$, permeability and capillary pressure as periodically oscillating functions by setting

$$
k_{j}(x, y, s)=\left\{\begin{array}{ll}
k_{j}^{+}(x, s) & \text { for } y \in[0,1) \\
k_{j}^{-}(x, s) & \text { for } y \in[-1,0)
\end{array}, \quad \tilde{p}_{c}(y, s)=\left\{\begin{array}{ll}
\tilde{p}_{c}^{+}(s) & \text { for } y \in[0,1) \\
\tilde{p}_{c}^{-}(s) & \text { for } y \in[-1,0)
\end{array} .\right.\right.
$$


Our aim is to investigate the non-degenerate limit problem of Definition 3.1 in this case. To simplify further, we assume that $p_{w, 0}(x)-p_{n, 0}(x)=\tilde{p}_{c}\left(x, y, s_{w, 0}(x, y)\right)$ holds as an equality in (3.7). This one-dimensional setting has been also studied in [16], where an effective system has been suggested by formal asymptotics, the correct effective system has been derived in [15], again by formal asymptotics, and it has been verified analytically in [26]. Regarding existence results and, in particular, interface conditions, we refer to $[7,12]$.

\subsection{Effective system in one space dimension}

Omitting once more the time dependence, the unknowns in (3.1)-(3.2) are $p_{j, 0}(x)$, $s_{j, 0}(x), p_{j, 1}(x, y), q_{j, 0}(x, y)$. Relation (3.5) implies that $q_{j, 0}(x, y)=q_{j, 0}(x)$ is independent of $y$. Furthermore, (3.7) yields that $s_{w, 0}(x, y)$ is independent of $y$ in the interval $(-1,0)$ and in the interval $(0,1)$. We can therefore abbreviate $s_{w, 0}(x, y)=s_{w, \pm}(x)=$ $s_{ \pm}(x)$ for $\pm y>0$, from (3.6) we know that the non-wetting saturation can be recovered as $s_{n, 0}(x, y)=s_{n, \pm}(x)=1-s_{ \pm}(x)$ for $\pm y>0$. Our last structural observation is that (3.4) yields for the effective pressure gradient $\partial_{x} p_{j, 0}(x)+\partial_{y} p_{j, 1}(x, y)=v_{j, \pm}(x)$ for $\pm y>0$. After these reductions, we have as unknowns

$$
\begin{aligned}
& p_{j, 0}(x) \text { and } s_{j, 0}(x):=f s_{j, 0}(x, y) d y \text { as macroscopic variables, and } \\
& s_{ \pm}(x), v_{j, \pm}(x) \text { as auxiliary variables describing microscopic properties. }
\end{aligned}
$$

In order to find a one-dimensional two-phase flow system, we have to express the six variables $s_{ \pm}(x)$ and $v_{j, \pm}(x)$ by the macroscopic variables. From the definition of $s_{j, 0}(x)$ and relation $(3.7)$ we obtain

$$
\begin{gathered}
s_{+}+s_{-}=2 s_{w, 0}(x), \\
\tilde{p}_{c}^{+}\left(s_{+}\right)=\tilde{p}_{c}^{-}\left(s_{-}\right) .
\end{gathered}
$$

Given $s_{w, 0}(x)$, this system can be solved for $s_{ \pm}=s_{ \pm}(x)$ due to the monotonicity of $\tilde{p}_{c}$. The facts that $p_{j, 1}(x, y)$ is periodic and that the left hand side in (3.4) is $y$-independent, imply

$$
\begin{aligned}
v_{j,+}+v_{j,-} & =2 \partial_{x} p_{j, 0}(x), \\
k_{j}^{+}\left(x, s_{j,+}(x)\right) v_{j,+} & =k_{j}^{-}\left(x, s_{j,-}(x)\right) v_{j,-} .
\end{aligned}
$$

Given $\partial_{x} p_{j, 0}(x)$, this system can be solved for $v_{j, \pm}=v_{j, \pm}(x)$ by positivity of the factors $k_{j}^{ \pm}$. We have thus seen that the six equations (5.1)-(5.4) determine the six auxiliary variables.

The macroscopic equations (3.3) can now be written in a compact form. We can reconstruct the saturations from the pressures with the two maps $\mathcal{S}_{w}$ and $\mathcal{S}_{n}$,

$$
\begin{aligned}
& \mathcal{S}_{j}: \Omega \times \mathbb{R}^{2} \rightarrow \mathbb{R}, \quad \mathcal{S}_{n}:=1-\mathcal{S}_{w}, \\
& \mathcal{S}_{w}:\left(x, p_{w}, p_{n}\right) \mapsto \frac{1}{2}\left(\tilde{p}_{c}^{+}(x, .)\right)^{-1}\left(p_{w}-p_{n}\right)+\frac{1}{2}\left(\tilde{p}_{c}^{-}(x, .)\right)^{-1}\left(p_{w}-p_{n}\right) .
\end{aligned}
$$


With the saturation $s_{w, 0}(x)=\mathcal{S}_{w}\left(x, p_{w}, p_{n}\right)$, we can define the flux functions as

$$
\begin{aligned}
\mathcal{F}_{j}: & \Omega \times \mathbb{R}^{2} \times \mathbb{R}^{2} \rightarrow \mathbb{R}, \\
\quad\left(x, p_{w}, p_{n}, \delta p_{w}, \delta p_{n}\right) & \mapsto k_{j}^{+}\left(x, s_{j,+}(x)\right) v_{j,+}
\end{aligned}
$$

where the quantities $s_{w, \pm}(x)=s_{ \pm}(x), s_{n, \pm}(x)=1-s_{w, \pm}(x)$ and $v_{j, \pm}(x)$ are calculated from (5.1)-(5.4) with $s_{w, 0}(x)=\mathcal{S}_{w}\left(x, p_{w}, p_{n}\right)$ and with $\partial_{x} p_{j, 0}(x)=\delta p_{j}$. The macroscopic effective two-phase flow equations $(3.3)$ for $\left(p_{w}(x, t), p_{n}(x, t)\right)$ now read

$$
\partial_{t} \mathcal{S}_{j}\left(x, p_{w}(x), p_{n}(x)\right)=\partial_{x} \mathcal{F}_{j}\left(x, p_{w}(x), p_{n}(x), \partial_{x} p_{w}(x), \partial_{x} p_{n}(x)\right)
$$

for $j=w$ and $j=n$.

The above reduction can be compared directly with the procedure in [26]. Our equations (5.1)-(5.2) coincide with equations (3.1) and (3.2) of [26], except that we write here $s_{ \pm}$instead of $u_{ \pm}$for saturation values. Our equation (5.3) relates to the geometric slope condition (3.6) of [26], our flux continuity (5.4) relates to the flux continuity (3.5) of [26]. In the contribution at hand we have chosen not to exploit the fact that the total flux is constant and given by the boundary condition. Instead, we stick with two effective equations, using the two indices $j=w$ and $j=n$, which makes the effective system more symmetric and more similar to the original system. As a consequence, we can avoid the additional unknowns $u_{ \pm, x}(x)$ of [26] in the set of microscopic variables.

\subsection{One-dimensional numerical experiment}

In order to validate the homogenized system (5.1)-(5.7), we consider the one dimensional trapping test problem introduced in section 4.1 of [16], and section 4 of [15]. The test problem investigates the permeabilities and capillary pressures

$$
k_{j}(x, y, s)=\left\{\begin{array}{ll}
k^{+} k_{r j}(s) & \text { for } y \in[0,1) \\
k^{-} k_{r j}(s) & \text { for } y \in[-1,0)
\end{array}, \quad \tilde{p}_{c}(y, s)= \begin{cases}\frac{1}{\sqrt{k^{+}}} J(s) & \text { for } y \in[0,1) \\
\frac{1}{\sqrt{k^{-}}} J(s) & \text { for } y \in[-1,0)\end{cases}\right.
$$

with

$$
k^{-}=0.5, \quad k^{+}=1.0, \quad k_{r w}(s)=(1-s)^{2}, \quad k_{r n}(s)=(s)^{2}, \quad J(s)=\frac{1}{\sqrt{1-s}},
$$

where $s$ now denotes the saturation of the non-wetting phase. We consider the problem in $\Omega=(-1,1)$ with initial saturation $s=1$, the total flux at the inflow boundary $(x=-1)$ equals one, and the saturation boundary datum at the inflow boundary is $s(-1)=0$ for all times.

For numerical purposes, we rewrite the two equations of (5.7) in a global flux formulation. Subtracting and adding the equations for both phases, we obtain

$$
2 \partial_{t} \mathcal{S}_{n}=\partial_{x}\left(\mathcal{F}_{n}-\mathcal{F}_{w}\right), \quad 0=\partial_{x}\left(\mathcal{F}_{n}+\mathcal{F}_{w}\right)
$$

The second equation of (5.8) yields the $x$-independence of the total flux. Since the total flux at the inflow is prescribed as 1, the second equation of (5.8) provides $\mathcal{F}_{n}+\mathcal{F}_{w}=1$. 


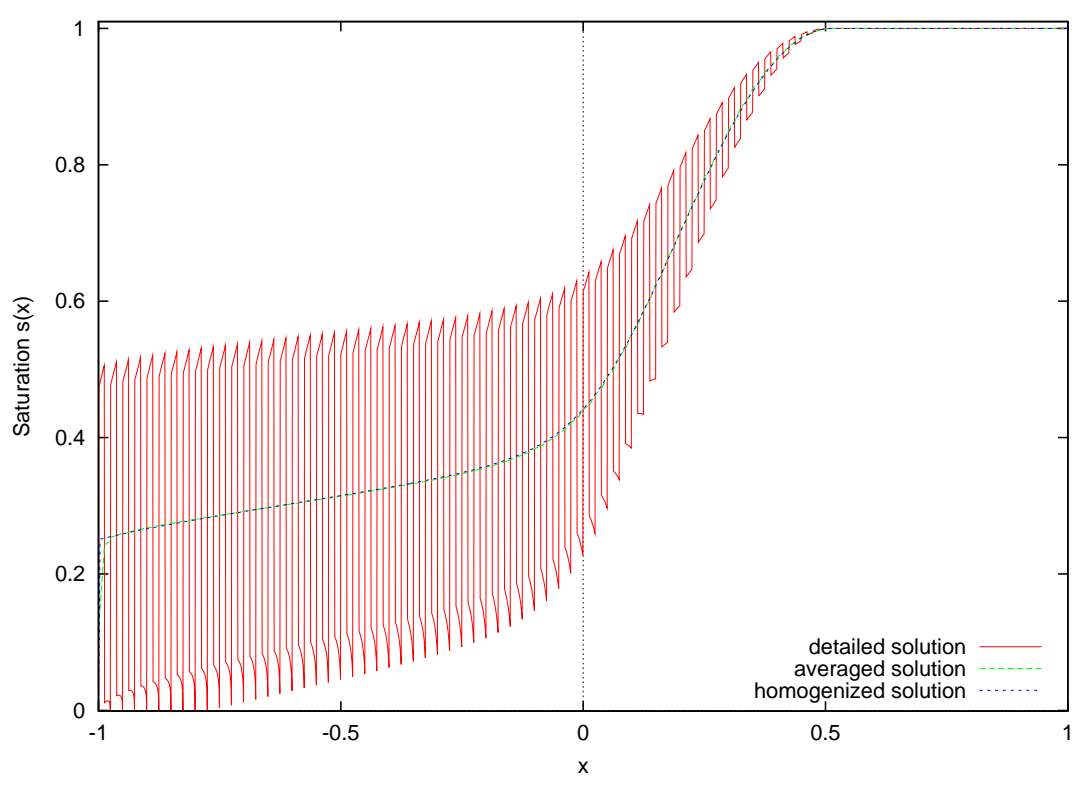

Figure 2: One-dimensional trapping effect. The graph shows saturation distributions at a fixed time instance. The solid line shows the result of a detailed simulation with 80 periodicity cells. The dashed line shows its averages over one period, the dotted line shows the solution of the homogenized problem.

It remains to solve the first equation of (5.8), the conservation law for the macroscopic saturation, where the fluxes are determined by (5.6). This homogenized global flux formulation coincides with the effective model that was derived formally in [15]. A formal distinction is that the definition of the effective macroscopic capillary pressure, given here through equation (5.5), is expressed in terms of the microscopic variables in [15]. However, taking into account (5.1)-(5.2), it is clear that both definitions coincide.

The numerical results shown in Figures 2 and 3 are obtained with an explicit upwind finite volume approximation of the homogenized system (5.1)-(5.7), based on the global flux formulation. Obviously, they are in agreement with the results for the effective model from [15]. In particular, Figure 2 illustrates the oil-trapping effect of the fine scale simulation with 80 periodic cells. The zoom in of Figure 3 shows a close to perfect matching between the numerical solution of the homogenized equation on a coarse grid with 640 grid cells with the cell-averaged fine scale simulation obtained on a fine grid with 3200 cells. In both cases grid convergence is ensured with the chosen mesh sizes.

We emphasize that we present here fine-scale simulations and calculations for the effective system, not the results of numerical multi-scale methods. The work at hand opens the way to investigate, in a direct comparison, numerical multi-scale methods for two-phase flow in porous media as developed, e.g., in [17, 18, 23]. 


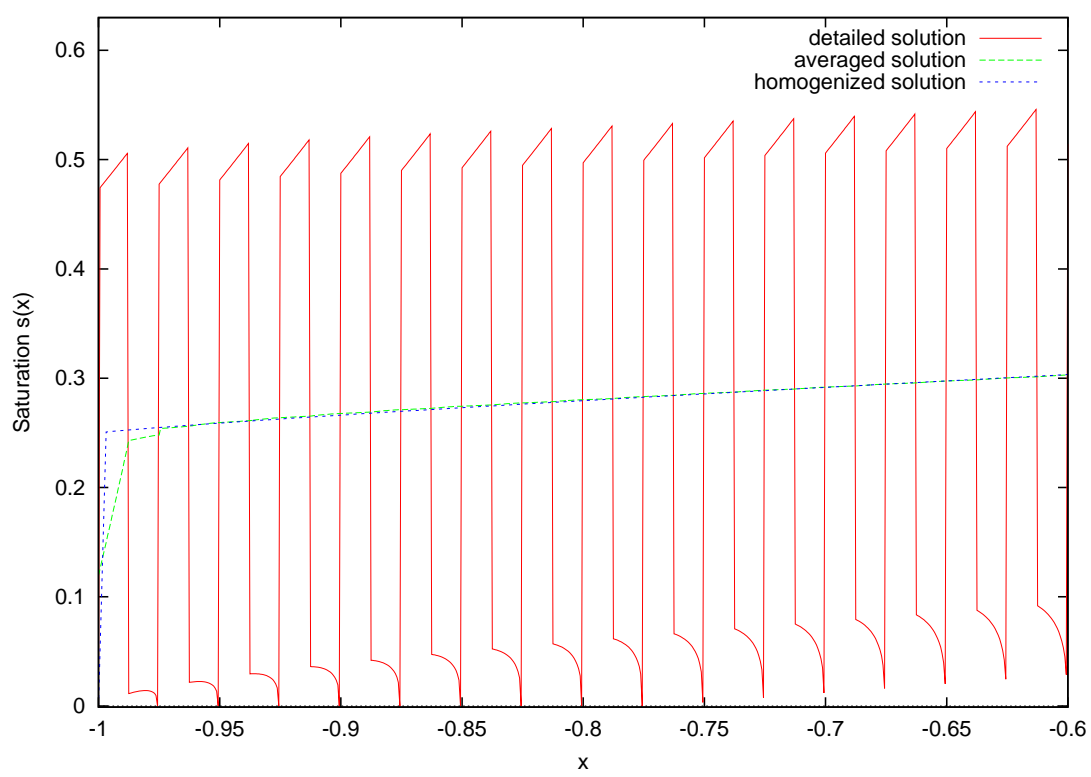

Figure 3: A zoom of the graphs of Figure 2.

\section{Conclusions}

Using the concept of weighted solutions, we have derived a rigorous homogenization result for degenerate two phase flow in porous media. Our result covers multiple space dimensions and the situation in which no scaling law is assumed between the local permeability values. Our contribution therefore closes a gap in the homogenization analysis of two phase flow in porous media that was left open in [28], where one of the local media coefficients was supposed to scale with $\varepsilon^{\varpi}, \varpi>0$. In the one dimensional case, the resulting homogenized system coincides with the effective model that was formally derived in [15] and rigorously obtained in [26]. In this sense, our result can be regarded as a generalization of [26] to the multi-dimensional case.

\section{Acknowledgement}

Support by DFG-grants SCHW 639/3-1 and OH 98/4-2 is gratefully acknowledged.

\section{References}

[1] G. Allaire. Homogenization and two-scale convergence. SIAM J. Math. Anal., 23(6):1482-1518, 1992.

[2] G. Allaire and F. Murat. Homogenization of the Neumann problem with nonisolated holes. Asymptotic Anal., 7(2):81-95, 1993. With an appendix written jointly with A. K. Nandakumar.

[3] H. Alt, S. Luckhaus, and A. Visintin. On nonstationary flow through porous media. Ann. Mat. Pura Appl. (4), 136:303-316, 1984. 
[4] H. W. Alt and E. DiBenedetto. Nonsteady flow of water and oil through inhomogeneous porous media. Ann. Scuola Norm. Sup. Pisa Cl. Sci. (4), 12(3):335-392, 1985.

[5] B. Amaziane, S. Antontsev, L. Pankratov, and A. Piatnitski. Homogenization of immiscible compressible two-phase flow in porous media: application to gas migration in a nuclear waste repository. Multiscale Model. Simul., 8(5):20232047, 2010.

[6] A. Beliaev. Homogenization of two-phase flows in porous media with hysteresis in the capillary relation. European J. Appl. Math., 14(1):61-84, 2003.

[7] M. Bertsch, R. Dal Passo, and C. J. v. Duijn. Analysis of oil trapping in porous media flow. SIAM J. Math. Anal., 35(1):245-267 (electronic), 2003.

[8] A. Bourgeat and A. Hidani. Effective model of two-phase flow in a porous medium made of different rock types. Appl. Anal., 58(1-2):1-29, 1995.

[9] A. Bourgeat, S. M. Kozlov, and A. Mikelić. Effective equations of two-phase flow in random media. Calc. Var. Partial Differential Equations, 3(3):385-406, 1995.

[10] A. Bourgeat, S. Luckhaus, and A. Mikelić. Convergence of the homogenization process for a double-porosity model of immiscible two-phase flow. SIAM J. Math. Anal., 27(6):1520-1543, 1996.

[11] A. Bourgeat and M. Panfilov. Effective two-phase flow through highly heterogeneous porous media: capillary nonequilibrium effects. Comput. Geosci., 2(3):191-215, 1998.

[12] F. Buzzi, M. Lenzinger, and B. Schweizer. Interface conditions for degenerate two-phase flow equations in one space dimension. Analysis (Munich), 29(3):299$316,2009$.

[13] Z. Chen. Degenerate two-phase incompressible flow. I. Existence, uniqueness and regularity of a weak solution. J. Differential Equations, 171(2):203-232, 2001.

[14] Z. Chen. Degenerate two-phase incompressible flow. II. Regularity, stability and stabilization. J. Differential Equations, 186(2):345-376, 2002.

[15] C. J. v. Duijn, H. Eichel, R. Helmig, and I. S. Pop. Effective equations for twophase flow in porous media: the effect of trapping at the micro scale. CASA report 2005-35, Eindhoven, 2005.

[16] C. J. v. Duijn, A. Mikelić, and I. S. Pop. Effective equations for two-phase flow with trapping on the micro scale. SIAM J. Appl. Math., 62(5):1531-1568 (electronic), 2002.

[17] P. Henning and M. Ohlberger. The heterogeneous multiscale finite element method for advection-diffusion problems with rapidly oscillating coefficients and large expected drift. Netw. Heterog. Media, 5(4):711-744, 2010. 
[18] P. Henning and M. Ohlberger. A-posteriori error estimation for a heterogeneous multiscale method for monotone operators and beyond a periodic setting. Preprint 01/11 - N, FB 10 , Universität Münster, 2011.

[19] J. Koch, A. Rätz, and B. Schweizer. Two-phase flow equations with a dynamic capillary pressure. Preprint TU Dortmund, 2011, submitted.

[20] D. Kröner and S. Luckhaus. Flow of oil and water in a porous medium. $J$. Differential Equations, 55(2):276-288, 1984.

[21] M. Lenzinger and B. Schweizer. Two-phase flow equations with outflow boundary conditions in the hydrophobic-hydrophilic case. Nonlinear Anal., 73(4):840-853, 2010 .

[22] D. Lukkassen, G. Nguetseng, and P. Wall. Two-scale convergence. Int. J. Pure Appl. Math., 2(1):35-86, 2002.

[23] M. Ohlberger. A posteriori error estimates for the heterogeneous multiscale finite element method for elliptic homogenization problems. Multiscale Model. Simul., 4(1):88-114 (electronic), 2005.

[24] M. Ohlberger and B. Schweizer. Modelling of interfaces in unsaturated porous media. Discrete Contin. Dyn. Syst., (Dynamical Systems and Differential Equations. Proceedings of the 6th AIMS International Conference, suppl.):794-803, 2007.

[25] B. Schweizer. Regularization of outflow problems in unsaturated porous media with dry regions. J. Differential Equations, 237(2):278-306, 2007.

[26] B. Schweizer. Homogenization of degenerate two-phase flow equations with oil trapping. SIAM J. Math. Anal., 39(6):1740-1763, 2008.

[27] B. Schweizer. The Richards equation with hysteresis and degenerate capillary pressure. J. Differential Equations, 252:5594-5612, 2012.

[28] L.-M. Yeh. Homogenization of two-phase flow in fractured media. Math. Models Methods Appl. Sci., 16(10):1627-1651, 2006.

[29] V. V. Zhikov. On two-scale convergence. Tr. Semin. im. I. G. Petrovskogo, (23):149-187, 410, 2003. 


\section{Preprints ab 2010/02}

2012-04

2012-03

2012-02

2012-01

2011-14

2011-13

2011-12

2011-11

2011-10

2011-09

2011-08

2011-07

2011-06

2011-05

2011-04

2011-03

2011-02
Patrick Henning, Mario Ohlberger, and Ben Schweizer

Homogenization of the degenerate two-phase flow equations

Andreas Rätz

A new diffuse-interface model for step flow in epitaxial growth

Andreas Rätz and Ben Schweizer

Hysteresis models and gravity fingering in porous media

Wilfried Hazod

Intrinsic topologies on H-contraction groups with applications to semistability

Guy Bouchitté and Ben Schweizer

Plasmonic waves allow perfect transmission through sub-wavelength metallic gratings

Waldemar Grundmann

Moment functions and Central Limit Theorem for Jacobi hypergroups on $[0, \infty[$

J. Koch, A. Rätz, and B. Schweizer

Two-phase flow equations with a dynamic capillary pressure

Michael Voit

Central limit theorems for hyperbolic spaces and Jacobi processes on $[0, \infty[$

Ben Schweizer

The Richards equation with hysteresis and degenerate capillary pressure

Andreas Rätz and Matthias Röger

Turing instabilities in a mathematical model for signaling networks

Matthias Röger and Reiner Schätzle

Control of the isoperimetric deficit by the Willmore deficit

Frank Klinker

Generalized duality for k-forms

Sebastian Aland, Andreas Rätz, Matthias Röger, and Axel Voigt

Buckling instability of viral capsides - a continuum approach

Wilfried Hazod

The concentration function problem for locally compact groups revisited: Non-dissipating space-time random walks, $\tau$-decomposable laws and their continuous time analogues

Wilfried Hazod, Katrin Kosfeld

Multiple decomposability of probabilities on contractible locally compact groups

Alexandra Monzner* and Frol Zapolsky $\dagger$

A comparison of symplectic homogenization and Calabi quasi-states

Stefan Jäschke, Karl Friedrich Siburg and Pavel A. Stoimenov

Modelling dependence of extreme events in energy markets using tail copulas 
The needle problem approach to non-periodic homogenization

A unifying approach to fractional Lévy processes

$2010-15$

Alexander Schnurr and Jeannette H.C. Woerner

Well-balanced Lévy Driven Ornstein-Uhlenbeck Processes

2010-14 Lorenz J. Schwachhöfer

On the Solvability of the Transvection group of Extrinsic

Symplectic Symmetric Spaces

2010-13 Marco Veneroni

Stochastic homogenization of subdifferential inclusions via scale integration

2010-12 Agnes Lamacz, Andreas Rätz, and Ben Schweizer

A well-posed hysteresis model for flows in porous media and

applications to fingering effects

2010-11 Luca Lussardi and Annibale Magni

$\Gamma$-limits of convolution functionals

2010-10 Patrick W. Dondl, Luca Mugnai, and Matthias Röger

Confined elastic curves

2010-09 Matthias Röger and Hendrik Weber

Tightness for a stochastic Allen-Cahn equation

2010-08 Michael Voit

Multidimensional Heisenberg convolutions and product formulas

for multivariate Laguerre polynomials

2010-07 Ben Schweizer

Instability of gravity wetting fronts for Richards equations with hysteresis

2010-06 Lorenz J. Schwachhöfer

Holonomy Groups and Algebras

2010-05 Agnes Lamacz

Dispersive effective models for waves in heterogeneous media

2010-04 Ben Schweizer and Marco Veneroni

Periodic homogenization of Prandtl-Reuss plasticity equations in arbitrary dimension

2010-03 Holger Dette and Karl Friedrich Siburg and Pavel A. Stoimenov

A copula-based nonparametric measure of regression dependence

2010-02 René L. Schilling and Alexander Schnurr

The Symbol Associated with the Solution of a Stochastic Differential Equation 